\title{
Rate Expressions in Mean Field Microkinetic Models Incorporating Multiple Types of Active Sites
}

\author{
Gregory Collinge ${ }^{a, g \star}$, Devyani Sharma ${ }^{f, g}$, Jean-Sabin McEwen ${ }^{a, b, c, d, e \star}$, and Mark \\ Saeys $^{\text {* }}$
}

a The Gene \& Linda Voiland School of Chemical Engineering and Bioengineering, Washington State

University, Pullman WA 99164, USA

${ }^{b}$ Department of Physics and Astronomy, Washington State University, Pullman, WA 99164, USA

c Department of Chemistry, Washington State University, Pullman, WA 99164, USA

d Institute for Integrated Catalysis, Pacific Northwest National Laboratory, Richland, WA, 99352, USA

e Department of Biological Systems Engineering, Washington State University, Pullman, WA 99164, USA

${ }^{f}$ Dutch Institute for Fundamental Energy Research (DIFFER), P.O. Box 6336, 5600 HH Eindhoven, The Netherlands

g Laboratory for Chemical Technology, Ghent University, Technologiepark 914, 9052 Gent, Belgium

* Corresponding authors:

Jean-Sabin McEwen; 509-335-8580 (phone), 509-335-4806 (fax), jis.mcewen@wsu.edu

Mark Saeys; +32 9-331-1754 (phone), mark.saeys@ugent.be

Gregory Collinge; 509-993-6086 (phone), gbcollinge@gmail.com

\begin{abstract}
It is well known that heterogenous catalysts exhibit a distribution of sites/structures, some more active than others but more than one often being important to the underlying reaction mechanism(s). The inclusion of this reality in mean field microkinetic models has been largely avoided in favor of lattice-based models like cluster expansions where in principle different types of sites can be explicitly defined. Here, we develop a thermodynamically self-consistent theory of multi-site microkinetics from the first principles of statistical mechanics to show how multiple site types can be represented in mean field microkinetic models. The theory incorporates local enthalpies and entropies, lateral molecular interactions, and global entropy; generating thermodynamic activities for any number of site types that deviate significant from those of idealized models. We provide the resultant rate expressions for rates of adsorption/desorption and surface diffusion between the site types. Contrary to what is typically assumed, even when a species has access to many different sites or binding configurations, only one rate, which is driven by the average adlayer chemical potential, can be defined for desorption from the surface. The approach in this work correctly describes adsorption/desorption and diffusion for a multisite model of a heterogenous catalyst and differs from the commonly used law of mass action.
\end{abstract}

\section{Introduction}

The development and use of microkinetic models is ubiquitous in heterogenous catalysis. ${ }^{1}$ However, few such models incorporate multiple types of potential binding sites. ${ }^{2-4}$ This is unsurprising since the work of Boudart ${ }^{5,6}$ on the kinetics of nonuniform surfaces (i.e., one with more than a single type of binding site) suggested that they should produce the same kinetics as uniform surfaces if one assumes a two-step catalytic reaction and a Boltzmann distribution of variable-affinity binding sites. Boudart's conclusions would therefore seem to obviate the need for multiple sites in microkinetic models. However, it is becoming increasingly clear that multiple active centers exist that are relevant to the kinetics of such systems, ${ }^{3,4,7-29}$ and recent work has highlighted the critical role of metastable state structures in heterogeneous catalysis. ${ }^{30-33}$ So, a proper formulation is still required.

Kinetic models with multiple site types (and other mathematically similar structures) have been rigorously formulated and explored in the work of Kreuzer and various co-workers. ${ }^{34}$ These models are generally built by treating the adlayer as an interacting, spatially-resolved lattice gas that is then inserted into the grand canonical partition function. The system's time-dependent properties can then 
be determined using transfer matrix ${ }^{35-37}$ or stochastic Monte Carlo (MC) methods ${ }^{38,39}$ depending on the level of complexity built into the model. While such models permit a detailed exploration into the microscopic origins of adsorption/desorption behavior, their complexity makes the modeling of large chemical reaction networks a largely intractable problem, whether including multiple site types or not. Despite the recent development and availability of powerful kinetic Monte Carlo (kMC) software, e.g., Zacros $^{40-43}$ for simulating these types of models, it is still clear that challenges remain for increasing the complexity of the systems one can study with such a methodology. ${ }^{44}$

If surface diffusion is fast compared to adsorption/desorption, the chemical adlayer can be assumed to be in quasi-equilibrium where spatial correlation between surface sites disappears. This allows one to project the complex space of adsorbate configurations onto a single set of collective variables: the areal coverages of each species adsorbed on the surface. In the cases where this assumption is valid (or valid enough to reasonable approximation), the adlayer can be described by its "mean field." This mean field is a single free energy value assigned to the entire adlayer, representing essentially the statistical average of the underlying spatially-resolved Hamiltonian at some constant set of adsorbate coverages, or as we will show, a constant set of adsorbate and site-type coverages. This allows one to describe all thermodynamic and kinetic properties as a function described as a function of macroscopic coverages alone, permitting the construction of mean-field microkinetic models (MFMKMs). To be certain, the lack of spatial correlation represents a significant limitation on such models, and other kinetic methodologies have been developed in an attempt to mitigate these limitations, ${ }^{45,} 46$ but it is clear that the utility and conceptual simplicity of standard MF-MKMs will continue to make them indispensable to researchers and industrial chemical engineers, alike. Therefore, it is important to determine how these models should properly incorporate multiple site types.

In this work, we derive MF-MKM rate expressions for site-to-site diffusion and adsorption/desorption that are valid when multiple site types are present. In the derivation of these rate expressions, we find it is both possible and necessary to explicitly define the otherwise implicit mean-field free energy of the adlayer. This quantity is central to ensuring what we will term thermodynamic self-consistency, which is an extension of thermodynamic consistency and detailed balance $^{34}$ that seeks to guarantee that equilibrium values, as predicted by minimization of the system's free energy, are reproduced when the MF-MKM is chemically isolated and allowed to come to equilibrium (i.e., when forward and reverse rates of reaction are equivalent). To enhance the physicality of the MF-MKM, its entropy contribution is highly generalized within a (mean-field) lattice gas paradigm where site blocking effects are fully incorporated analytically. The forms of the resulting rate expressions are found to deviate considerably from those typically assumed unless one treats all adsorbates as two-dimensional ideal gases (i.e., non-interacting free translators). Nonetheless, their analytical nature ensures they can be easily implemented in any MF-MKM.

\section{Theory and Formulation}

\subsection{Assumptions and Model Setup}

When we speak of site types on a surface, the terms can be broadly applied. In fact, the mathematical formulation presented here is equally applicable to any sort of "deposition" of any molecular entity into any n-dimensional space. For instance, instead of "coverage" of adsorbates, concentration of species inside a bulk solid or even just inside empty 3D space could be used. Additionally, the adsorbate could simply occupy multiple chemical states instead of spatially distinct sites, in which case rates of diffusion can be interpreted equivalently as rates of conversion from one state to another. This is illustrated in Figure 1, where we use red circles and blue triangles to represent site types and/or states, with diffusion or conversion represented by purple curved arrows. Thus, while we will continue referring to them as "sites" and "site types", it should not be construed as a limitation of the model. Due to this generalization, even amorphous surfaces/spaces like step defected surfaces or multi-faceted nanoparticles can be modeled provided spatial correlation can be sufficiently averaged out to produce a valid mean field. While we will restrict ourselves to the case of a single molecular species (the "adsorbates") occupying a surface, the results are equally valid if multiple molecular species are present. 
a. Model Representation

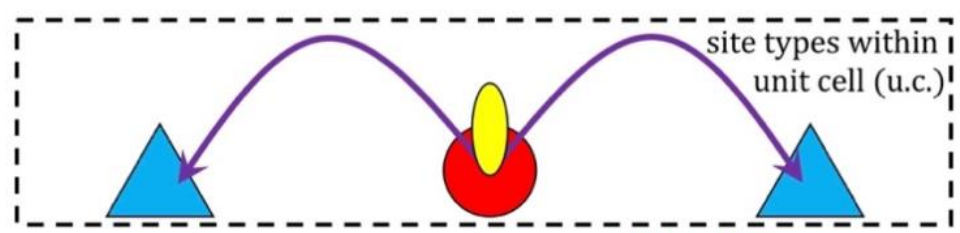

b. Representing Diffusion from Site to Site

e.g. O diffusion on an $\mathrm{FCC}(111)$ surface from top to hollow sites

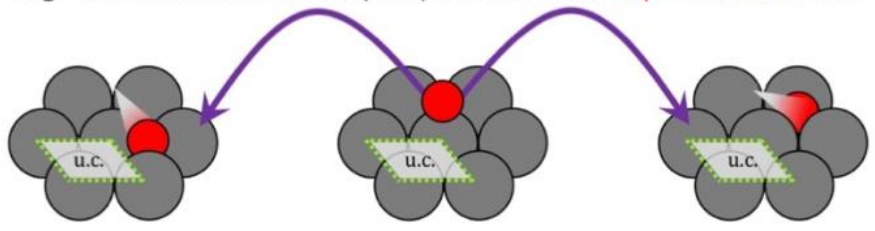

c. Representing Conversion from State to State

e.g. Keto-enol tautomerization of adsorbed phenol

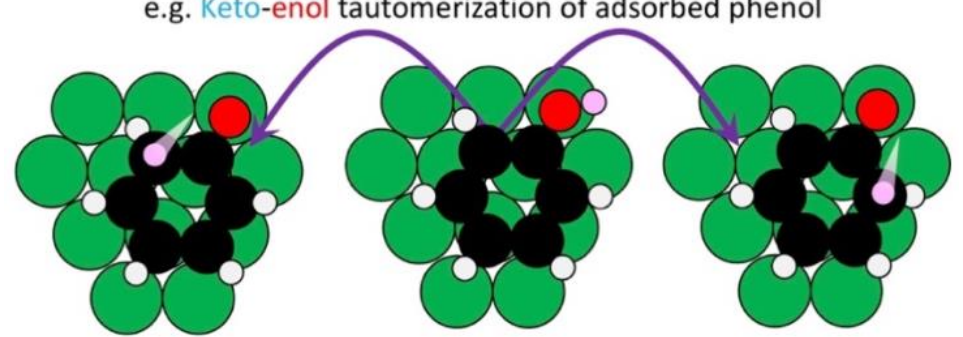

Figure 1. Illustration of how (a) the "site types" (red circles or blue triangles) in our model and "diffusion" of an adsorbate (yellow oval) can be interpreted as both (b) diffusion from site-to-site, such as oxygen adatom diffusion from top to hollow sites on an FCC(111) surface or (c) conversion from state-to-state, such as the keto-enol tautomerization of phenol on the FCC(111) surface. In (b) and (c), red spheres are $\mathrm{O}$ atoms, black spheres are $\mathrm{C}$ atoms, white spheres are $\mathrm{H}$ atoms, and light pink spheres are $\mathrm{H}$ atoms colored different to aid the eye. The grey and green spheres are both the FCC(111) top surface layer, colored different to enhance contrast with the adsorbates. Purple arrows represent the movement needed to create diffusion or conversion.

With this in mind, we first take a surface made up of a very large but finite number of unit cells (u.c.), $\mathrm{N}_{\text {u.c. }}$, onto which some number of adsorbates of a single species, $\mathrm{N}$, are adsorbed. Defining unit

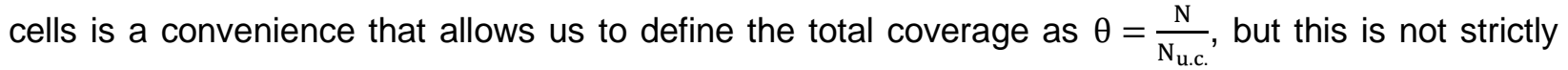
required-total surface area and thus surface density could be defined instead. On this surface is some number of total adsorption sites with multiple potential site types. The site types will be superscripted with $A, B, C$, etc. (leaving subscripts available for molecular identity) and the number of each site type per unit cell is denoted as $n^{(A)}, n^{(B)}, n^{(C)}$, etc.; which, in the case where sites are not homogenously distributed, can be computed as $\frac{\mathrm{N}_{\mathrm{S}}^{(\mathrm{X})}}{\mathrm{N}_{\text {u.c. }}}$ where $\mathrm{N}_{\mathrm{S}}^{(\mathrm{X})}$ is the total number of $\mathrm{X}$ sites on the entire surface. A simple example of potential site types are the top, bridge, and hollow sites of $\operatorname{FCC}(111)$ surfaces (where $\mathrm{n}^{\text {(top) }}=1, \mathrm{n}^{\text {(bridge) }}=3$, and $\mathrm{n}^{\text {(hollow) }}=2$ ). Our goal is to determine the proper expression for rates of diffusion from one site type to another and for adsorption/desorption when a variety of such site types are occupied.

We define rates according to transition state theory, so that rates of diffusion from, e.g., site type $X$ to site type $\mathrm{Y}$, and rate of adsorption/desorption from, e.g., an $\mathrm{X}$ site to some reservoir, are given by:

$$
\begin{aligned}
& r_{X \rightarrow Y}=\frac{k_{B} T}{h} \frac{1}{q_{\text {r.c. }}^{\ddagger}} e^{-\beta\left[\mu_{X Y}^{\ddagger}-\mu_{X \rightarrow Y}\right]} \\
& r_{X \rightarrow r e s}=\frac{k_{B} T}{h} \frac{1}{q_{\text {r.c. }}^{\ddagger}} e^{-\beta\left[\mu_{X-r e s}^{\ddagger}-\mu_{X} \rightarrow \text { res }\right]}
\end{aligned}
$$

where $r_{X \rightarrow Y}$ is rate of diffusion from site type $X$ to site type $Y, r_{X \rightarrow r e s}$ is the rate of desorption from site type $\mathrm{X}$ to some reservoir appropriate to the adsorbate and system in question; $\mathrm{k}_{\mathrm{B}}$ and $\mathrm{h}$ are the 
Boltzmann and Planck constants, respectively; $\mathrm{T}$ is the absolute temperature; $\mathrm{q}_{\mathrm{r} \text {.c. }}^{\ddagger}$ is the partition function of the transition state associated with movement along the reaction coordinate (usually taken to be that of an ideal 1D gas or an imaginary vibrational mode); $\beta=\left(\mathrm{k}_{\mathrm{B}} \mathrm{T}\right)^{-1}$ is the thermodynamic beta; $\mu_{\mathrm{XY}}^{\ddagger}$ and $\mu_{\mathrm{X}-\mathrm{res}}^{\ddagger}$ are the chemical potential of the transition state complex associated with diffusion from site type $X$ to site type $Y$ and the chemical potential associated with the desorption (or adsorption) of adsorbates from site type $\mathrm{X}$ to the appropriate reservoir (e.g. the gas phase), respectively; and $\mu_{X \rightarrow Y}$ and $\mu_{\mathrm{X} \rightarrow \mathrm{res}}$ are the corresponding chemical potentials associated with the driving force for diffusion and desorption, respectively, whose functional forms we make no assumptions about a priori. This therefore means we make no assumption about the functional form of reactants' chemical activities either, which are generally taken to be equal to their molecular concentrations according to the law of mass action. We will show that these "driving force chemical potentials" are not path dependent as implied by the " $\mathrm{X} \rightarrow \mathrm{Y}$ " in $\mu_{\mathrm{X} \rightarrow \mathrm{Y}}$, but this presumption is not made here to ensure we do not bias our derivation to that result. Regardless, it will be the elucidation of these chemical potentials that ultimately defines these rates.

Site-to-site diffusion is neglected in mean-field microkinetic models because it is inconsequential to the (free) energy of the system. That is, if the coverage of adsorbates is not changed by diffusion, which is the case in single-site models, the average energy of the adsorbates do not change eitherthey are all moving through the same mean field. However, when multiple sites are present on the surface, the coverages of the site types will indeed change upon diffusion from site type to site type. This means that this type of diffusion is in fact of consequence even in a mean field model-because the mean field is changed. We show this in Figure 2, which illustrates how even if diffusion is from one unit cell to a nearby one, it only matters if the adsorbate changes site type. This also implies that only the adsorbates occupying the same types of sites must be assumed to be in quasi-equilibrium instead of the entire adlayer in a multi-site mean field model. The consequence of this will be shown in Section 3.

\section{A.) A-to-A Diffusion}

$$
\theta^{(\mathrm{A})} \text { const. } \mid \theta^{(\mathrm{B})} \text { const. }
$$

$\Rightarrow \mathrm{r}_{\mathrm{A} \rightarrow \mathrm{A}}$ is inconsequential
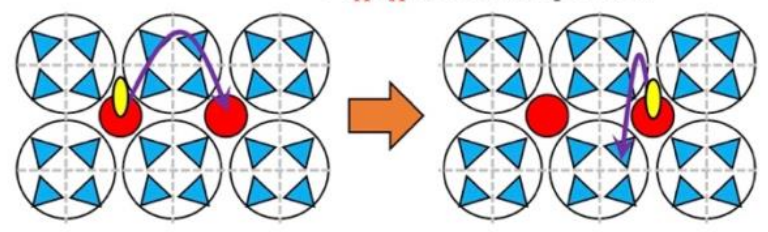

D.) B-to-A Diffusion $\theta^{(A)}$ incr. $\mid \theta^{(B)}$ decr.
$r_{B \rightarrow A}=\frac{k_{B} T}{h} e^{-\beta\left[\mu_{A B}^{ \pm}-\mu_{B \rightarrow A}\right]}$
B.) A-to-B Diffusion $\theta^{(A)}$ decr. $\mid \theta^{(B)}$ incr. $r_{A \rightarrow B}=\frac{k_{B} T}{h} e^{-\beta\left[\mu_{A B}^{ \pm}-\mu_{A \rightarrow B}\right]}$

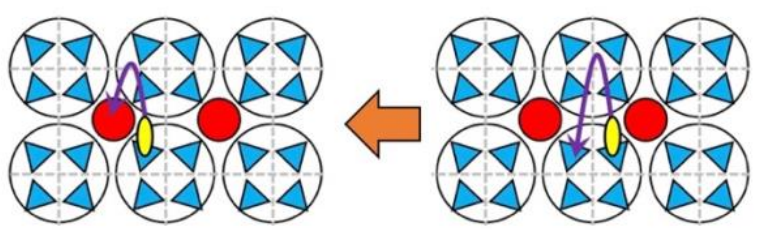

C.) B-to-B Diffusion

$\theta^{(B)}$ const. $\mid \theta^{(A)}$ const.

$\Rightarrow \mathrm{r}_{\mathrm{B} \rightarrow \mathrm{B}}$ is inconsequential

Figure 2. Illustration of how site-to-site diffusion between the same types of sites ( $A$ and $C$ ) and different types of sites ( $B$ and $D$ ) effect mean-field microkinetic rates. In the illustrations, each large open circle is a supposed surface atom with the surface unit cell delineated by dashed grey lines. As in Figure 1, red circles and blue triangles are site types $A$ and $B$, respectively.

\subsection{Derivation of Chemical Potentials}


To determine the chemical potentials in Equation 1, our strategy will be to ensure what we term "thermodynamic self-consistency": the state of the system at equilibrium, according to equality of rate expressions, should match that predicted by equilibrium statistical mechanics. Simply put, this means we will require that when all rates of diffusion and rates of adsorption/desorption (Equation $1 \mathrm{a}$ and 1b, respectively) reach the same value, the same equilibrium is reached that one finds using the partition function of the same system. Such a requirement obviously requires that one have access to the system's global partition function. We start constructing this by first defining the total free energy of a single configuration, $\boldsymbol{\sigma}$, of adsorbates. To model this free energy, we will use a mean-field treatment of a lattice gas Hamiltonian ${ }^{38,47-49}$, which is itself a sum over occupied sites that accounts for the presence of lateral interactions and includes the local partition function of each occupied site.

$$
\mathrm{F}_{\boldsymbol{\sigma}}=\left[\sum_{\mathrm{X}}^{\text {site types }} \mathrm{N}^{(\mathrm{X})}\left(\mathrm{V}^{(\mathrm{X})}-\mathrm{k}_{\mathrm{B}} \mathrm{T} \ln \left[\mathrm{q}^{(\mathrm{X})}\right]\right)\right]+\mathrm{N}_{\text {u.c. }} \mathrm{V}_{\text {lat }}
$$

where $\mathrm{N}^{(\mathrm{X})}$ is the total number of adsorbates occupying site type $\mathrm{X}$, such that $\sum_{\mathrm{X}} \mathrm{N}^{(\mathrm{X})}=\mathrm{N}$ is the total number of (same molecular identity) adsorbates on the surface; $\mathrm{V}^{(\mathrm{X})}$ is the binding energy of an isolated adsorbate bound to an $\mathrm{X}$ site; $\mathrm{q}^{(\mathrm{X})}$ is its local molecular partition function describing its thermally accessible degrees of freedom within a single $X$ site; and $V_{\text {lat }}$ is a term (with units of energy per unit cell here) that accounts for non-ideality and typically takes the form of a multinomial expansion of site coverages in a mean-field treatment of a lattice gas. $F_{\sigma}$ is an extensive variable as written, so it will be useful to normalize the free energy on the number of unit cells to give an intensive variable:

$$
\mathrm{F}_{\text {u.c. }}=\frac{\mathrm{F}_{\boldsymbol{\sigma}}}{\mathrm{N}_{\text {u.c. }}}=\left[\sum_{\mathrm{X}}^{\text {site types }} \theta^{(\mathrm{X})}\left(\mathrm{V}^{(\mathrm{X})}-\mathrm{k}_{\mathrm{B}} \mathrm{T} \ln \left[\mathrm{q}^{(\mathrm{X})}\right]\right)\right]+\mathrm{V}_{\text {lat }}
$$

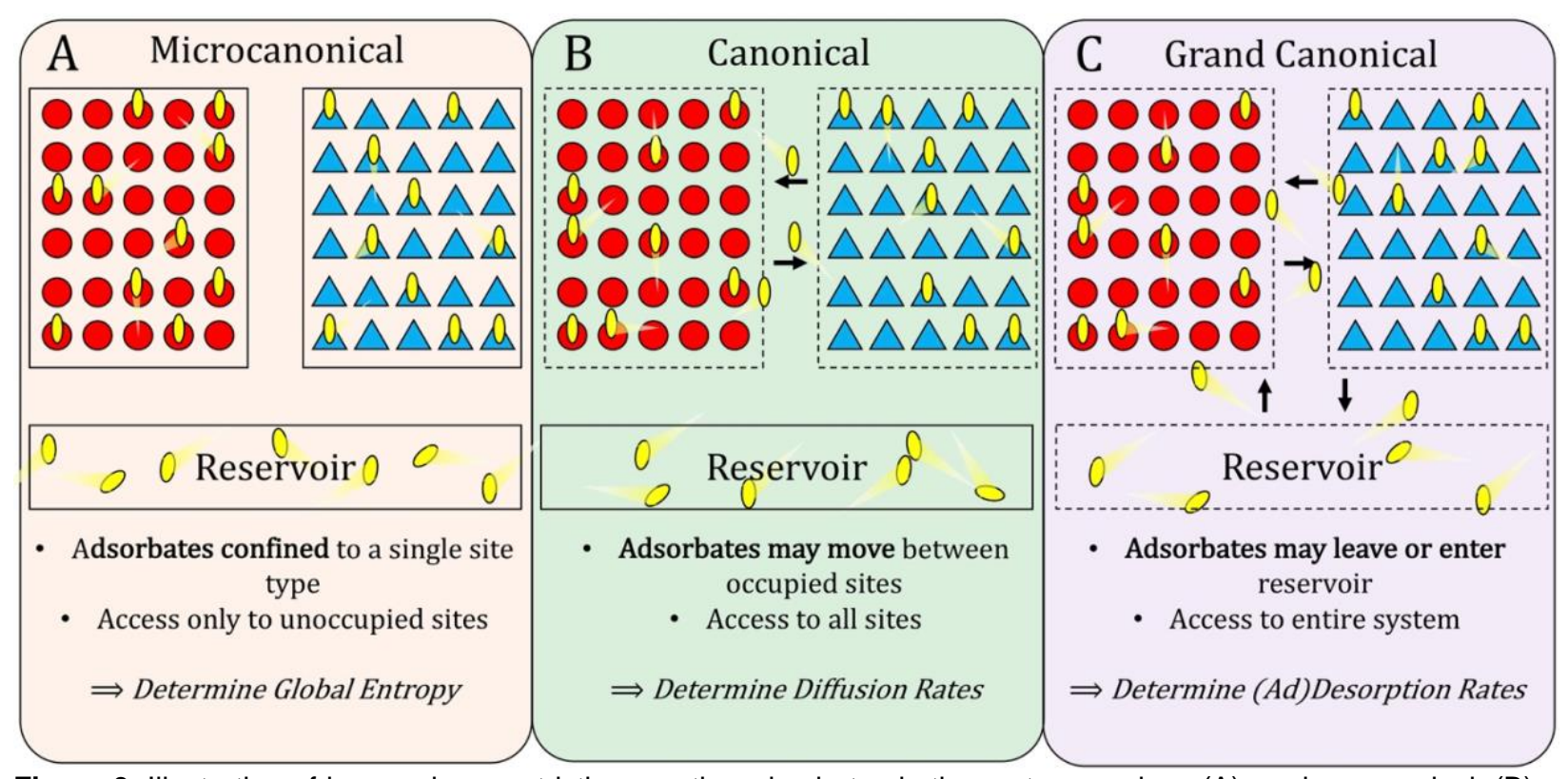

Figure 3. Illustration of how various restrictions on the adsorbates in the system produce $(A)$ a microcanonical, (B) a canonical, and (C) a grand canonical ensemble, with each building off the next in terms of complexity. The shapes shown are the same as those used in Figure 1 and Figure 2. The topmost boxes containing sites with no adsorbates present represent all available empty sites of the site type contained within while the middle boxes with adsorbates present represent all occupied sites. The bottommost box is, as labeled, a reservoir where the adsorbates reside when outside the surface. Dashed borders around each box represents permeability and arrows represent where exchanges are permitted. 
which also allows us to formally define $\theta^{(X)}=\frac{N^{(X)}}{N_{u c}}$ as denoting the coverage of adsorbates occupying $\mathrm{X}$ sites. Coverages are thus defined on a per unit cell basis, ensuring that the basis for defining coverage is never ambiguous even when there are different numbers of adsorption sites per unit cell. It is not a requirement that the coverages be bounded between 0 and 1 . We should note that it is common to assume that $\mathrm{q}^{(\mathrm{X})}$ is that of a harmonic oscillator, which is independent of coverage. However, in general, $\mathrm{q}^{(\mathrm{X})}$ could be found using an anharmonic model or made coverage dependent to capture further non-idealities.

Equations 2 and 3 describe a local free energy that would define a local partition function for a single adlayer configuration. However, in any system at equilibrium, all of its properties will be described by its global partition function, which is a thermodynamic description of the entire system that includes all possible adlayer configurations. We must therefore define the underlying global partition function. To do this, we work in stages: first considering the micro-canonical case, then the canonical case, and finally the grand canonical case. This is graphically illustrated in Figure 3.

\subsubsection{Microcanonical Ensemble}

If the coverages of a species bound to one of its available site types, $\theta^{(A)}, \theta^{(B)}, \ldots, \theta^{(X)}$, are kept fixed, the (mean-field) energy of the system will stay fixed regardless of the configuration the adsorbates take on the surface. Even if we account for the local degrees of freedom as in Equation 2, any temperature used will map to a unique local free energy (i.e., $\mathrm{F}_{\text {u.c. }}$ ) due to the fixing of coverages. Thus, since the surface size, number of adsorbates, and energy of the system are kept fixed, we are nominally dealing with a microcanonical ensemble. We can define the canonical partition function corresponding to this microcanonical ensemble by explicitly including the constant energy of the ensemble in it, which will be useful in the next steps. This produces the "microcanonical" $(\mu \mathrm{c})$ partition function, $Q_{\mu c}$, as:

$$
\mathrm{Q}_{\mu \mathrm{c}}=\Omega \mathrm{e}^{-\beta \mathrm{F}_{\sigma}}
$$

where $\Omega$ is the number of degenerate energy states, dependent upon the total number of adsorbates on the surface. Note that $\Omega$ is in fact the "true" microcanonical partition function here, and, as we will see, is the source of the adsorbates' global entropy and thus chemical activities. We will return to the derivation and functional forms of $\Omega$ in Section 2.3. For now, however, we borrow the result from that section that this term can always be written as

$$
\Omega=\prod_{\mathrm{X}}\left[\omega^{(\mathrm{X})}\right]^{\mathrm{N}_{\mathrm{u} . c .}}
$$

where $\omega^{(\mathrm{X})}$ is the contribution to the number of degenerate energy states from adsorbates occupying site type X. Since all the terms in Equation 4 depend on variables that are kept fixed here, the free energy of this microcanonical ensemble is directly determinable as

$$
\begin{aligned}
& \mathrm{F}_{\mu \mathrm{c}}=-\mathrm{k}_{\mathrm{B}} \mathrm{T} \ln \left(\mathrm{Q}_{\mu \mathrm{c}}\right)=\mathrm{F}_{\sigma}-\mathrm{k}_{\mathrm{B}} \mathrm{T} \ln (\Omega) \\
& \Rightarrow \mathrm{F}_{\mu \mathrm{c}}=\mathrm{N}_{\text {u.c. }}\left[\mathrm{F}_{\text {u.c. }}-\mathrm{k}_{\mathrm{B}} \mathrm{T} \sum_{\mathrm{X}} \ln \left(\omega^{(\mathrm{X})}\right)\right]
\end{aligned}
$$

and this gives its global entropy as

$$
\mathrm{S}_{\mu \mathrm{c}}=\left(\mathrm{k}_{\mathrm{B}} \mathrm{N}_{\text {u.c. }} \sum_{\mathrm{X}}^{\text {site types }}\left[\theta^{(\mathrm{X})}\left(\ln \left(\mathrm{q}^{(\mathrm{X})}\right)+\mathrm{T} \frac{\partial \ln \left(\mathrm{q}^{(\mathrm{X})}\right)}{\partial \mathrm{T}}\right)\right]\right)+\left(\mathrm{N}_{\text {u.c. }} \sum_{\mathrm{X}}^{\text {site types }} \mathrm{k}_{\mathrm{B}} \ln \left(\omega^{(\mathrm{X})}\right)\right)
$$

where the first term in parentheses is the sum of local entropies arising from thermally-induced fluctuations of each bound adsorbate; while the second term, involving $\omega^{(\mathrm{X})}$, corresponds to the only source of non-thermal global entropy, specifically resulting from the configurational space spanned by the adsorbates on the entire surface.

\subsubsection{Canonical Ensemble}


We now allow adsorbates to move from one site type to any of the other site types present but continue to keep the total number of adsorbates on the surface fixed. This is shown in Figure 3B. While the total number of adsorbates, $\mathrm{N}$, does not change, the population of occupied site types, $\mathrm{N}^{(\mathrm{X})}$, does. Since this can result in a change in the local free energy (see Equation 2), we are now working with a canonical ensemble. We will assume three site types, A, B, and C, are present, which will provide the minimal requirements to generalize the result at the end. The canonical partition function, $\mathrm{Q}_{\text {canon }}$, is then given as

$$
\begin{aligned}
Q_{\text {canon }}= & \sum_{N^{(B)}=0}^{N-N^{(A)}} \sum_{N^{(A)}=0}^{N} Q_{\mu c}=\sum_{N^{(B)}=0}^{N-N^{(A)}} \sum_{N^{(A)}=0}^{N} e^{-\beta F_{\mu c}} \\
& =\sum_{N^{(B)}=0}^{N-N^{(A)}} \sum_{N^{(A)}=0}^{N}\left[\omega^{(A)} \omega^{(B)} \omega^{(C)} e^{-\beta F_{u} \text {.c. }}\right]^{N_{\text {u.c. }} .}
\end{aligned}
$$

where the constraint that $\mathrm{N}^{(\mathrm{A})}+\mathrm{N}^{(\mathrm{B})}+\mathrm{N}^{(\mathrm{C})}=\mathrm{N}$ is implicitly imposed, and we have factored $\mathrm{N}_{\text {u.c. }}$ out of each term to create a single exponent. Since $\mathrm{N}$ is held constant, only two of the three site type occupations can be independently defined, i.e., there are only two independent variables, which is reflected in the presence of only two summations. These are $\mathrm{N}^{(\mathrm{A})}$ and $\mathrm{N}^{(\mathrm{B})}$ in Equation 7, but any two could be arbitrarily chosen (which will become an important element in the next section). In general, there will always be $\mathrm{k}-1$ independent $\mathrm{N}^{(\mathrm{X})}$ variables given $\mathrm{k}$ total site types.

In the thermodynamic limit, $\mathrm{N}_{\text {u.c. }}$ approaches infinity, and it is therefore appropriate to perform a change of variables from discrete values (e.g., number of adsorbates populating site type $\mathrm{X}, \mathrm{N}^{(\mathrm{X})}$ ) to continuous values (fractional coverage of site type $\mathrm{X}, \theta^{(\mathrm{X})}$ ) via

$$
\mathrm{N}^{(\mathrm{X})}=\theta^{(\mathrm{X})} \mathrm{N}_{\text {u.c. }}
$$

which allows the summations in Equation 7 to be replaced with integrals

$$
Q_{\text {canon }}=N_{\text {u.c. }}^{2} \int_{0}^{\theta_{\max }^{(B)}-\theta^{(A)} \theta_{\max }^{(A)}} \int_{0}^{(A)}\left[\omega^{(B)} \omega^{(C)} e^{-\beta F_{u . c .}}\right]^{N_{\text {u.c. }}} \mathrm{d} \theta^{(A)} d \theta^{(B)}
$$

where we account for the fact that maximum coverages, $\theta_{\max }^{(\mathrm{A})}$ and $\theta_{\max }^{(\mathrm{B})}$ (relative to the fixed total coverage), may be some value other than unity. In the thermodynamic limit where $\mathrm{N}_{\text {u.c. }}$ approaches infinity, the integrand in Equation $8 \mathrm{~b}$ becomes heavily peaked around the maximum value of $\left[\omega^{(\mathrm{A})} \omega^{(\mathrm{B})} \omega^{(\mathrm{C})} \mathrm{e}^{-\beta \mathrm{F}_{\text {u.c. }}}\right.$. . This is due to the exponential dependence on $\mathrm{N}_{\text {u.c. }}$ which exaggerates the maximum value to the point where the integrand becomes a Dirac delta. That is, as $\mathrm{N}_{\text {u.c. }}$ approaches infinity, the value that maximizes $\left[\omega^{(\mathrm{A})} \omega^{(\mathrm{B})} \omega^{(\mathrm{C})} \mathrm{e}^{-\beta \mathrm{F}_{\mathrm{u} . c} .}\right]$ will be the only value that gets integrated, making this value equivalent to $Q_{\text {canon. }}$. That is,

$$
\mathrm{Q}_{\text {canon }}=\mathrm{N}_{\text {u.c. }}^{2}\left[\max \left(\mathrm{e}^{-\beta \mathrm{F}_{\mu \mathrm{c}}}\right)\right]
$$

where we have collected the $\omega_{\mathrm{X}}$ terms back into the exponent. Maximizing the integrand of Equation $8 \mathrm{~b}$ requires that the critical points of the integrand be met as suggested by Equation $8 \mathrm{C}$. This establishes the minimum criteria for equilibrium in a canonical ensemble where a fixed number of adsorbates can move freely between sites. Specifically, the critical points can be found by solving

$$
\begin{aligned}
& \frac{\partial}{\partial \theta^{(A)}}\left(N_{\text {u.c. }}^{2}\left[\omega^{(A)} \omega^{(B)} \omega^{(C)} e^{-\beta F_{u . c .}}\right]^{N_{\text {u.c. }}}\right)_{\theta, \theta^{(B)}}=0, \quad d \theta^{(A)}=-d \theta^{(C)} \\
& \frac{\partial}{\partial \theta^{(B)}}\left(N_{\text {u.c. }}^{2}\left[\omega^{(A)} \omega^{(B)} \omega^{(C)} e^{-\beta F_{\text {u.c. }}}\right]^{N_{\text {u.c. }}}\right)_{\theta, \theta(A)}=0, \quad d \theta^{(B)}=-d \theta^{(C)}
\end{aligned}
$$

where we have noted, on the right-hand side, the differential relationship that arises due to the counterbalance between the dependent variable $\left(\theta^{(\mathrm{C})}\right)$ and the independent variables $\left(\theta^{(\mathrm{A})}, \theta^{(\mathrm{B})}\right)$ through $\theta^{(A)}+\theta^{(B)}+\theta^{(C)}=\theta$. After application of the chain rule, Equations $9 \mathrm{a}$ and $9 \mathrm{~b}$ reduce to

$$
\left(\frac{\partial \mathrm{F}_{\mu \mathrm{c}}}{\partial \theta^{(\mathrm{A})}}\right)_{\theta, \theta^{(\mathrm{B})}}=0, \quad \mathrm{~d} \theta^{(\mathrm{A})}=-\mathrm{d} \theta^{(\mathrm{C})}
$$




$$
\left(\frac{\partial \mathrm{F}_{\mu \mathrm{c}}}{\partial \theta^{(\mathrm{B})}}\right)_{\theta, \theta^{(\mathrm{A})}}=0, \quad \mathrm{~d} \theta^{(\mathrm{B})}=-\mathrm{d} \theta^{(\mathrm{C})}
$$

where we have substituted the results from Equation 6a into Equation 9a and 9b, and again noted the relationships among differentials.

These critical points comprise the basis for ensuring thermodynamic self-consistency within this formulation. At equilibrium, when all rates are equal to one another, these critical points must be satisfied. Equation $9 \mathrm{c}$ indicates that when the coverage of $A$ sites differentially increases while keeping the coverage of $B$ sites and total coverage constant, the differential change in free energy resulting from this increase and the concomitant decrease in coverage of $C$ sites must be zero at equilibrium. In order to keep the coverage of B sites and total coverage constant, an adsorbate must implicitly move from a $\mathrm{C}$ site to an $\mathrm{A}$ site (reflected in the relationship between differentials shown). This suggests that the thermodynamic driving force for diffusion between $A$ and $C$ sites should vanish when the system reaches specifically the critical point in Equation 9c, while that for diffusion between $A$ and $C$ sites should vanish when the system reaches the critical point in Equation 9d. We can make this mathematically explicit by recasting the differentials in Equations $9 \mathrm{c}$ and $9 \mathrm{~d}$ in terms of chemical potentials of adsorbates occupying the various site types (we show the derivation in Section S1 of the $\mathrm{SI})$. This gives that

$$
\begin{aligned}
& \left(\frac{\partial \mathrm{F}_{\mu \mathrm{c}}}{\partial \theta^{(\mathrm{A})}}\right)_{\theta, \theta^{(B)}}=\mathrm{N}_{\text {u.c. }}\left[\left(\frac{\partial \mathrm{F}_{\mu \mathrm{c}}}{\partial \mathrm{N}^{(\mathrm{A})}}\right)_{\mathrm{N}^{(\mathrm{B})}, \mathrm{N}^{(\mathrm{C})}}-\left(\frac{\partial \mathrm{F}_{\mu \mathrm{c}}}{\partial \mathrm{N}^{(\mathrm{C})}}\right)_{\mathrm{N}^{(\mathrm{A})}, \mathrm{N}^{(\mathrm{B})}}\right]=\mathrm{N}_{\text {u.c. }}\left[\mu^{(\mathrm{A})}-\mu^{(\mathrm{C})}\right] \\
& \left(\frac{\partial \mathrm{F}_{\mu \mathrm{c}}}{\partial \theta^{(\mathrm{B})}}\right)_{\theta, \theta^{(\mathrm{A})}}=\mathrm{N}_{\text {u.c. }}\left[\left(\frac{\partial \mathrm{F}_{\mu \mathrm{c}}}{\partial \mathrm{N}^{(\mathrm{B})}}\right)_{\mathrm{N}^{(\mathrm{A})}, \mathrm{N}^{(\mathrm{C})}}-\left(\frac{\partial \mathrm{F}_{\mu \mathrm{c}}}{\partial \mathrm{N}^{(\mathrm{C})}}\right)_{\mathrm{N}^{(\mathrm{A})}, \mathrm{N}^{(\mathrm{B})}}\right]=\mathrm{N}_{\text {u.c. }}\left[\mu^{(\mathrm{B})}-\mu^{(\mathrm{C})}\right]
\end{aligned}
$$

where careful attention to subscripts on the differentials is required. Recalling that $\mathrm{F}_{\mu \mathrm{c}}$ is indeed the extensive canonical free energy, we are able to identify the differentials in square brackets (center expression) as the usual chemical potentials (the right hand side) corresponding the addition of one adsorbate to the system at a specific site type while keeping all others constant. Inserting Equations $10 \mathrm{a}$ and $10 \mathrm{~b}$ into Equations $9 \mathrm{c}$ and $9 \mathrm{~d}$, respectively, gives that each critical point is respectively satisfied when

$$
\begin{aligned}
& \mu^{(A)}=\mu^{(C)} \\
& \mu^{(B)}=\mu^{(C)}
\end{aligned}
$$

which will be exactly met if the rates of diffusion from $A$ to $C$ sites (and vice versa) and $B$ to $C$ sites (and vice versa) are defined as:

$$
\begin{aligned}
& \mathrm{r}_{\mathrm{A} \rightarrow \mathrm{C}}=\frac{\mathrm{k}_{\mathrm{B}} \mathrm{T}}{\mathrm{h}} \frac{1}{q_{r . c .}^{\ddagger}} \mathrm{e}^{-\beta\left[\mu_{\mathrm{AC}}^{\ddagger}-\mu^{(\mathrm{A})}\right]} \text { and } \mathrm{r}_{\mathrm{C} \rightarrow \mathrm{A}}=\frac{\mathrm{k}_{\mathrm{B}} \mathrm{T}}{\mathrm{h}} \frac{1}{q_{r . c .}^{\ddagger}} \mathrm{e}^{-\beta\left[\mu_{\mathrm{AC}}^{\ddagger}-\mu^{(\mathrm{C})}\right]} \\
& \mathrm{r}_{\mathrm{B} \rightarrow \mathrm{C}}=\frac{\mathrm{k}_{\mathrm{B}} \mathrm{T}}{\mathrm{h}} \frac{1}{q_{r . c .}^{\ddagger}} \mathrm{e}^{-\beta\left[\mu_{\mathrm{BC}}^{\ddagger}-\mu^{(\mathrm{B})}\right]} \text { and } \mathrm{r}_{\mathrm{C} \rightarrow \mathrm{B}}=\frac{\mathrm{k}_{\mathrm{B}} \mathrm{T}}{\mathrm{h}} \frac{1}{q_{r . c .}^{\ddagger}} \mathrm{e}^{-\beta\left[\mu_{\mathrm{BC}}^{\ddagger}-\mu^{(\mathrm{C})}\right]}
\end{aligned}
$$

where we can now see that $r_{X \rightarrow Y}$ depends solely on the chemical potential of $\mu^{(X)}$ with the only term unique to the specific pathway connecting sites $X$ and $Y$ being the chemical potential of the transition state: $\mu_{\mathrm{XY}}^{\ddagger}$. This result is perhaps unsurprising since requirements for equilibrium are defined by the equality of chemical potentials. However, since we have now shown that the free energy of the system can be defined explicitly as in Equation 6a, its partial differentials can be explicitly assessed. Performing this differentiation to get, as an example, $\mu^{(\mathrm{A})}$, produces

$$
\begin{gathered}
\mu^{(A)}=\left(\frac{\partial F_{\mu c}}{\partial N^{(A)}}\right)_{N^{(B)}, N^{(C)}}=\mu^{(A 1)}+\mu^{(A 2)}+\mu^{(A 3)} \\
\mu^{(A 1)}=V^{(A)}+\frac{\partial V_{\text {lat }}}{\partial \theta^{(A)}} \\
\mu^{(A 2)}=-k_{B} T\left[\ln \left(q^{(A)}\right)+\sum_{X}^{\text {site types }}\left(\theta^{(X)} \frac{\partial \ln \left(q^{(X)}\right)}{\partial \theta^{(A)}}\right)\right]
\end{gathered}
$$




$$
\mu^{(\mathrm{A} 3)}=-\mathrm{k}_{\mathrm{B}} \mathrm{T}\left[\sum_{\mathrm{X}}^{\text {site types }} \frac{\partial \ln \left(\omega^{(\mathrm{X})}\right)}{\partial \theta^{(\mathrm{A})}}\right]
$$

where $\mu^{(\mathrm{A} 1)}, \mu^{(\mathrm{A} 2)}$, and $\mu^{(\mathrm{A} 3)}$ represent contributions, respectively, from the mean-field electronic ground state energy; from the thermal part of the local free energy; and from the non-thermal global entropy, which we will show manifests as adsorbate chemical activity in rate expressions. If $\mathrm{q}^{(\mathrm{X})}$ in Equation $13 \mathrm{c}$ does not exhibit coverage dependence in any way, $\mu^{(\mathrm{A} 2)}$ reduces to simply $-\mathrm{k}_{\mathrm{B}} \mathrm{T} \ln \left(\mathrm{q}^{(\mathrm{A})}\right)$, but if one wishes to incorporate a coverage dependence, the full set of differentials would need to be assessed.

Note that rates corresponding to diffusion between A and B sites are conspicuously absent from Equation $12 \mathrm{a}$ and $12 \mathrm{~b}$. These rates are established by observing that we could have equally chosen to make either the coverage of $A$ sites or the coverage of $B$ sites, $\theta^{(A)}$ or $\theta^{(B)}$, the dependent variable in Equation 8b; making $\theta^{(\mathrm{C})}$ the dependent variable was arbitrary. As we demonstrate in Section $\mathrm{S} 2$ of the $\mathrm{SI}$, this means we will always be able to match up each possible rate pair to exactly one unique critical point regardless of the number of site types invoked.

\subsubsection{Grand Canonical Ensemble}

Finally, we allow the system to permit exchange of adsorbates with a reservoir kept at some constant chemical potential, which we will denote as $\mu_{\text {res }}$, illustrated graphically in Figure $3 \mathbf{C}$. This is expanding the previous canonical ensemble into a grand canonical ensemble. The grand canonical partition function, $\Xi$, is thus

$$
\Xi=\sum_{N=0}^{N_{\max }} \sum_{N^{(B)}=0}^{N-N^{(A)}} \sum_{N^{(A)}=0}^{N} Q_{\mu c} e^{\beta \mu_{\text {res }} N}
$$

where the new summation runs over the total number of adsorbates on the surface up to some maximum, $\mathrm{N}_{\text {max }}$, allowed by the surface.

As before, we wish to operate in the thermodynamic limit $\left(\mathrm{N}_{\text {u.c. }} \rightarrow \infty\right)$ where variables become continuous. That is, we define

$$
\mathrm{N}=\theta \mathrm{N}_{\text {u.c. }}
$$

to create total coverage, $\theta$, as a continuous variable. However, if total coverage changes, the site coverages, $\theta^{(\mathrm{X})}$, must necessarily change in some way as well, making them inappropriate independent variables in conjunction with $\theta$. We circumvent this by defining site factions, $\mathrm{y}^{(\mathrm{X})}$, as the fraction of adsorbates present that occupy each site type:

$$
\theta^{(\mathrm{X})} \mathrm{N}_{\text {u.c. }}=\mathrm{y}^{(\mathrm{X})} \theta \mathrm{N}_{\text {u.c. }}
$$

Each site fraction runs from 0 to 1, inclusively, and all possible site-type coverages can be found within this domain regardless of the total coverage. Substituting Equations $15 \mathrm{a}$ and $15 \mathrm{~b}$ and their differentials into Equation 16 and converting the summations to integrals, produces

$$
\Xi=\int_{0}^{\theta_{\max }} \int_{0}^{1-y^{(A)}} \int_{0}^{1} N_{\text {u.c. }}^{3} \theta^{2}\left[\omega^{(A)} \omega^{(B)} \omega^{(C)} e^{-\beta\left(F_{u . c .}-\mu_{\text {res }} \theta\right)}\right]^{N_{u . c .}} d y^{(A)} d y{ }^{(B)} d \theta
$$

which is once again heavily peaked around the maximum value of the integrand. We note that $\theta_{\max }$ is the absolute maximum coverage allowed on the surface, and the definition of site fractions obviates the need for determining the relative maximum coverages for each site type. As in the canonical case, we can thus achieve the minimum requirements for equilibrium by satisfying the critical points of the integrand. After differentiating with respect to the each of the three independent variables in Equation 16 (while holding all others constant), we produce 


$$
\begin{aligned}
& \frac{\partial}{\partial y^{(A)}}\left(N_{\text {u.c. }}^{3} \theta^{2}\left[e^{-\beta\left(F_{\mu c}-\mu_{\text {res }} N_{\text {u.c. }} \theta\right)}\right]\right)_{y^{(B)}, \theta}=0 \\
& \Rightarrow \mathrm{N}_{\text {u.c. }}^{3} \theta \mathrm{e}^{\mu_{\text {res }} \mathrm{N}_{\text {u.c. }} \theta}\left[\frac{\partial}{\partial \theta^{(\mathrm{A})}}\left(\mathrm{e}^{-\beta \mathrm{F}_{\mu c}}\right)_{\theta^{(\mathrm{B})}, \theta}\right]=0 \\
& \Longrightarrow\left(\frac{\partial \mathrm{F}_{\mu \mathrm{c}}}{\partial \theta^{(\mathrm{A})}}\right)_{\theta, \theta^{(\mathrm{B})}}=0, \quad \mathrm{~d} \theta^{(\mathrm{A})}=-\mathrm{d} \theta^{(\mathrm{C})} \\
& \frac{\partial}{\partial y^{(B)}}\left(N_{\text {u.c. }}^{3} \theta^{2}\left[\mathrm{e}^{-\beta\left(F_{\mu c}-\mu_{\text {res }} N_{\text {u.c. }} \theta\right)}\right]\right)_{y^{(A)}, \theta}=0 \\
& \Rightarrow \mathrm{N}_{\text {u.c. }}^{3} \theta \mathrm{e}^{\mu_{\text {res }} N_{\text {u.c. }} \theta}\left[\frac{\partial}{\partial \theta^{(B)}}\left(\mathrm{e}^{-\beta \mathrm{F}_{\mu c}}\right)_{\theta}{ }^{(\mathrm{A})}, \theta\right]=0 \\
& \Rightarrow\left(\frac{\partial \mathrm{F}_{\mu \mathrm{c}}}{\partial \theta^{(\mathrm{B})}}\right)_{\theta, \theta^{(\mathrm{A})}}=0, \quad \mathrm{~d} \theta^{(\mathrm{B})}=-\mathrm{d} \theta^{(\mathrm{C})} \\
& \frac{\partial}{\partial \theta}\left(\mathrm{N}_{\text {u.c. }}^{3} \theta^{2}\left[\mathrm{e}^{-\beta\left(\mathrm{F}_{\mu c}-\mu_{\text {res }} \mathrm{N}_{\text {u.c. }} \theta\right)}\right]\right)_{\mathrm{y}^{(\mathrm{A})}, \mathrm{y}^{(B)}}=0 \\
& \Rightarrow\left[\frac{\partial}{\partial \mathrm{N}} \mathrm{N}^{2}\left(\left[\mathrm{e}^{-\beta\left(\mathrm{F}_{\mu \mathrm{c}}-\mu_{\mathrm{res}} \mathrm{N}\right)}\right]\right)_{\mathrm{y}(\mathrm{A}), \mathrm{y}^{(B)}}\right]=0 \\
& \Rightarrow\left(\frac{\partial \mathrm{F}_{\mu \mathrm{c}}}{\partial \mathrm{N}}\right)_{\mathrm{y}(\mathrm{A}), \mathrm{y}(\mathrm{B})}=\left\{\frac{2 \mathrm{k}_{\mathrm{B}} \mathrm{T}}{\mathrm{N}_{\text {u.c. }} \theta}\right\}+\mu_{\text {res }}, \quad \mathrm{dN}^{(\mathrm{X})}=\mathrm{y}^{(\mathrm{X})} \mathrm{dN}
\end{aligned}
$$

where we have again combined all $\omega^{(\mathrm{X})}$ into the exponent to convert $\mathrm{F}_{\text {u.c. }}$ to $\mathrm{F}_{\mu \mathrm{c}}$ and differential relationships that exist due to site balance are again shown to the right of each critical point. We have taken advantage of the fact that $\theta$ is constant in Equations $17 \mathrm{a}$ and $17 \mathrm{~b}$ to show that these two critical points are identical to those already found in the canonical case (Equations $9 \mathrm{c}$ and $9 \mathrm{~d}$ ). Thus, meeting these critical points is still a necessary condition to attain full equilibrium in the open system, and the results from Section 2.2.2 still apply. However, the critical point in Equation 17c is new, resulting from the introduction of total coverage, $\theta$, as an independent variable. The presence of the term in curly brackets makes this critical point seemingly odd, too, as it depends on both temperature and total coverage. This term is a finite size effect artifact: any finitely sized surface would need to account for this term. Thankfully, however, we operate in the thermodynamic limit, where this term approaches zero as $\mathrm{N}_{\text {u.c. }}$ approaches infinity at any finite temperature, giving

$$
\left(\frac{\partial \mathrm{F}_{\mu \mathrm{c}}}{\partial \mathrm{N}}\right)_{\mathrm{y}^{(\mathrm{A})}, \mathrm{y}^{(\mathrm{B})}, \mathrm{y}^{(\mathrm{C})}}=\mu_{\mathrm{res}}, \quad \mathrm{dN}^{(\mathrm{X})}=\mathrm{y}^{(\mathrm{X})} \mathrm{dN}
$$

which says that the differential addition of another adsorbate to the system occurring at constant fractional occupation of site types must equal the chemical potential of the adsorbate reservoir at equilibrium (since $\mathrm{y}^{(\mathrm{A})}+\mathrm{y}^{(\mathrm{B})}+\mathrm{y}^{(\mathrm{C})}=1$, one cannot hold $\mathrm{y}^{(\mathrm{A})}$ and $\mathrm{y}^{(\mathrm{B})}$ constant without also holding $\mathrm{y}^{(C)}$ constant as well). Since all $\mathrm{y}^{(\mathrm{X})}$ are held constant, and $\mathrm{N}^{(\mathrm{A})}+\mathrm{N}^{(\mathrm{B})}+\mathrm{N}^{(\mathrm{C})}=\mathrm{N}$, we arrive at the differential relationships shown to the right in Equation 17d. These differential relationships are the only way to satisfy the restriction that all $\mathrm{y}^{(\mathrm{X})}$ remain constant and essentially says that adsorption/desorption events do not directly perturb the fractional occupation of site types in a multisite mean-field model. This analysis also implies that since there's only one total coverage term per molecular species, there can only ever be one critical point, and therefore pair of rates, associated with adsorption/desorption of that species regardless of how many site types are present on the surface. This is an important result because it is tempting to assume that each site type exhibits its own desorption rate independent of the other rates of desorption, yet this analysis clearly shows that one cannot have this and ensure thermodynamic self-consistency at the same time. We must therefore recast the rate expression corresponding to adsorption/desorption as that of a general surface-toreservoir event, giving 


$$
\begin{gathered}
r_{\text {surf } \rightarrow \text { res }}=\frac{\mathrm{k}_{\mathrm{B}} \mathrm{T}}{\mathrm{h}} \frac{1}{\mathrm{q}_{\text {r.c. }}^{\ddagger}} \mathrm{e}^{-\beta\left[\mu_{\text {surf-res }}^{\ddagger}-\left(\frac{\partial \mathrm{F}_{\mu \mathrm{c}}}{\partial \mathrm{N}}\right)_{\mathrm{y}}(\mathrm{A}), \mathrm{y}^{(\mathrm{B})}, \mathrm{y}(\mathrm{C})\right]} \\
\mathrm{r}_{\text {res } \rightarrow \text { surf }}=\frac{\mathrm{k}_{\mathrm{B}} \mathrm{T}}{\mathrm{h}} \frac{1}{\mathrm{q}_{\text {r.c. }}^{\ddagger}} \mathrm{e}^{-\beta\left[\mu_{\text {surf-res }}^{\ddagger}-\mu_{\text {res }}\right]}
\end{gathered}
$$

which satisfies Equation 17d when $r_{\text {surf } \rightarrow \text { res }}=r_{\text {res } \rightarrow \text { surf }}$. Note that we have replaced the presumed individual transition states chemical potential, $\mu_{\mathrm{X}-\mathrm{res}}^{\ddagger}$, for adsorption/desorption from each site with a common transition state chemical potential, $\mu_{\text {surf-res }}^{\ddagger}$, that connects the surface and reservoir. We will return to the interpretation of $\mu_{\text {surf-res }}^{\ddagger}$ in the Discussion section. Nonetheless, we can see that $\left(\frac{\partial \mathrm{F}_{\mu \mathrm{c}}}{\partial \mathrm{N}}\right)_{\mathrm{y}^{(\mathrm{A})}, \mathrm{y}^{(\mathrm{B})}, \mathrm{y}^{(\mathrm{C})}}$ in Equation 18a is effectively a desorption chemical potential for the entire surface of $\mathrm{N}$ adsorbates. While its analytical expression can be found by differentiating Equation 6a with respect to the total number of adsorbates, it is easier and more instructive to determine its relationship to the site type chemical potentials. Using the same procedure used in Section 2.2.2 (see Section S1 of the $\mathrm{SI})$, we can recast this partial derivative as

$$
\begin{aligned}
&\left(\frac{\partial \mathrm{F}_{\mu \mathrm{c}}}{\partial \mathrm{N}}\right)_{\mathrm{y}^{(\mathrm{A})}, \mathrm{y}^{(\mathrm{B})}, \mathrm{y}^{(\mathrm{C})}} \mathrm{y}^{(\mathrm{A})}\left(\frac{\partial \mathrm{F}_{\mu \mathrm{c}}}{\partial \mathrm{N}^{(\mathrm{A})}}\right)_{\mathrm{N}^{(\mathrm{B})}, \mathrm{N}^{(\mathrm{C})}}+\mathrm{y}^{(\mathrm{B})}\left(\frac{\partial \mathrm{F}_{\mu \mathrm{c}}}{\partial \mathrm{N}^{(B)}}\right)_{\mathrm{N}^{(\mathrm{A})}, \mathrm{N}^{(\mathrm{C})}}+\mathrm{y}^{(\mathrm{C})}\left(\frac{\partial \mathrm{F}_{\mu \mathrm{c}}}{\partial \mathrm{N}^{(\mathrm{C})}}\right)_{\mathrm{N}^{(\mathrm{A})}, \mathrm{N}^{(B)}} \\
& \Rightarrow\left(\frac{\partial \mathrm{F}_{\mu \mathrm{c}}}{\partial \mathrm{N}}\right)_{\mathrm{y}^{(\mathrm{A})}, \mathrm{y}^{(\mathrm{B})}, \mathrm{y}^{(\mathrm{C})}}=\mathrm{y}^{(\mathrm{A})} \mu^{(\mathrm{A})}+\mathrm{y}^{(\mathrm{B})} \mu^{(\mathrm{B})}+\mathrm{y}^{(\mathrm{C})} \mu^{(\mathrm{C})}
\end{aligned}
$$

where again attention to subscripts on the differentials is required. This gives the general result that for an adsorbate of any single species, in a k-site model, its desorption chemical potential is given by

$$
\Rightarrow\left(\frac{\partial \mathrm{F}}{\partial \mathrm{N}}\right)_{\text {all } \mathrm{y}^{(\mathrm{x})}}=\sum_{\mathrm{X}}^{\text {site types }} \mathrm{y}^{(\mathrm{X})} \mu^{(\mathrm{X})}
$$

where we have dropped the " $\mu c$ " from the subscript of $F_{\mu c}$ since it is now clear that this expression is the mean field free energy of the adlayer regardless of which ensemble is being probed. We can see via inspection of Equations $19 \mathrm{a}$ or $19 \mathrm{~b}$ that when diffusion rates reach equilibrium according to Equation 10 (i.e., when $\mu^{(\mathrm{A})}=\mu^{(\mathrm{B})}=\mu^{(\mathrm{C})}=\cdots=\mu^{(\mathrm{X})}=\mu^{(\mathrm{eq})}$ ), the desorption chemical potential becomes exactly the equilibrated chemical potential, $\mu^{(\mathrm{eq})}$. Since $\sum_{\mathrm{X}}^{\text {site types }} \mathrm{y}^{(\mathrm{X})}=1$, Equation $19 \mathrm{~b}$ reduces to $\sum_{\mathrm{X}}^{\text {site types }} \mathrm{y}^{(\mathrm{X})} \mu^{(\mathrm{eq})}=\mu^{(\mathrm{eq})}$, which is precisely as designed. We will return to the rationale and implications of desorption rates taking the form shown in Equation 19a in the Discussion section.

\subsection{Derivation of Mean-Field Global Entropy, $\Omega$}

We now return to the definition of $\Omega$ in Equations 4 and 5 . This term represents the total number of configurations $\mathrm{N}$ adsorbates can take on a surface made up on $\mathrm{N}_{\text {u.c. }}$ unit cells and $\mathrm{n}^{(\mathrm{X})}$ sites of type $\mathrm{X}$ per unit cell. Since $\Omega$ is defined in the microcanonical case of Section 2.2.1, the total number of adsorbates, $\mathrm{N}$, and occupation of site types, $\mathrm{N}^{(\mathrm{X})}$, can be treated as constant. As a result, the number of configurations that $\mathrm{N}^{(\mathrm{X})}$ adsorbates can take, $\Omega^{(\mathrm{X})}$, need only account for the sites that are currently blocked by adsorbates at non-X sites. This makes the number of configurations, $\Omega^{(\mathrm{X})}$, that $\mathrm{N}^{(\mathrm{X})}$ adsorbates can take independent of the number of configurations, $\Omega^{(\mathrm{Y})}$, that $\mathrm{N}^{(\mathrm{Y})}$ adsorbates can simultaneously take. The site $X$-bound adsorbates have access to the remaining unblocked surface area. This means $\Omega$ can be written as a product of combinatorics for each site, i.e.,

$$
\Omega=\Omega^{(\mathrm{A})} \Omega^{(\mathrm{B})} \ldots \Omega^{(\mathrm{X})} \ldots
$$

In a mean field treatment of these configurations, any restrictions on site occupation due to the presence of other adsorbates (i.e., lateral interactions) are averaged out and expressed in the lateral 
interaction term, $\mathrm{V}_{\mathrm{lat}}$, shown in Equation 2. However, if adsorbates completely block other adsorbates from co-adsorbing nearby, that particular interaction would have to be treated as an infinitely repulsive lateral interaction term that cannot be subsumed into $V_{\text {lat }}$ (the average of any set of values that contains an infinite term is infinity). A similar argument exists for finite but very large lateral interactions. As a result, this type of site blocking has to be accounted for in each $\Omega^{(\mathrm{X})}$ of Equation 20.

As already mentioned, $\Omega$ will be shown to control the chemical activity of adsorbates. To show the importance of this, we consider first what $\Omega$ is for a 2D gas/free translator ("Case 0"), the simplest possible model, incorporating no site blocking effects or restriction on movement/diffusion. We will prove that this is the model tacitly assumed in most MF-MKMs in the literature. We will then look at four possible more-realistic site-blocking cases due to co-adsorption: (I) mutually exclusive coadsorption, (II) self-exclusive co-adsorption, (III) self-inclusive co-adsorption, and (IV) mutually inclusive co-adsorption. These cases are illustrated in Figure 4. Sterling's approximation:

is used throughout.

$$
\mathrm{N} !=\mathrm{N}^{\mathrm{N}} \mathrm{e}^{-\mathrm{N}}
$$

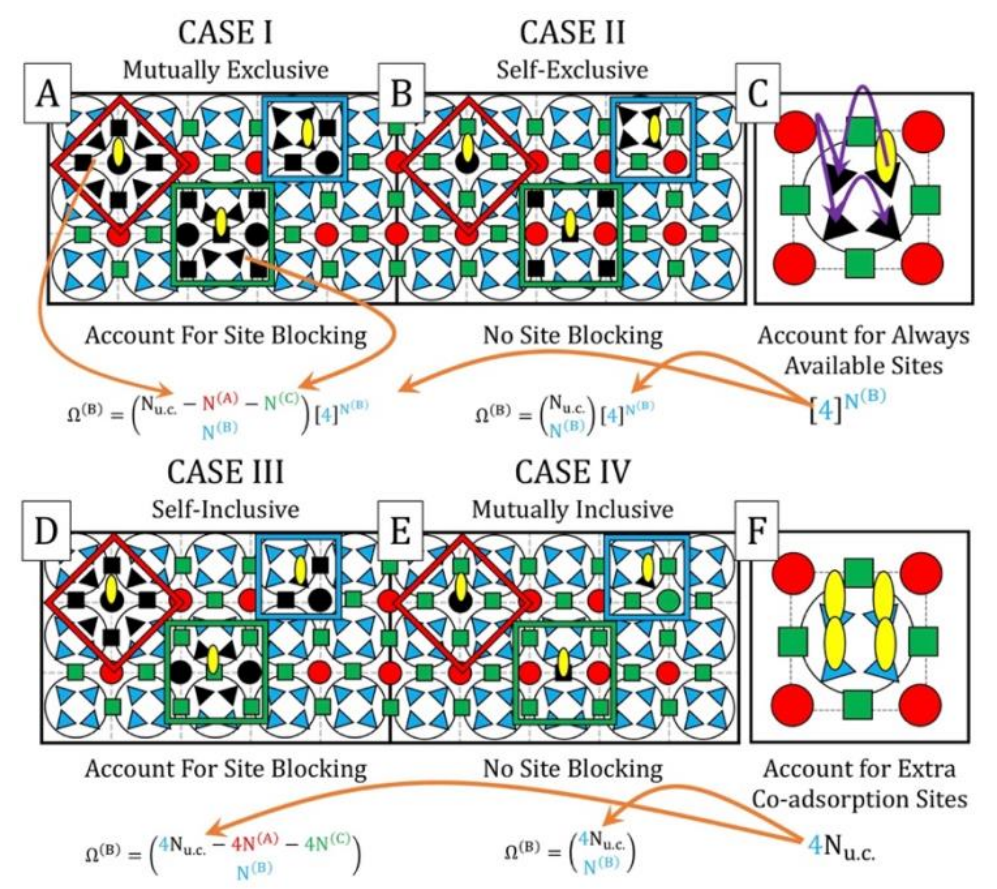

Figure 4. Different site-blocking cases for co-adsorption of a molecular species. Available A sites, B sites, and C sites are represented by red circles, blue triangles, and green squares, respectively. Blocked sites are colored in black. Here, $\Omega^{(B)}$ is defined as an example, and the numbers of $A$ sites, $B$ site, and $C$ sites per unit cell are: $n^{(A)}=1, n^{(B)}=4$, and $\mathrm{n}^{(\mathrm{C})}=2$. Cases I and II, $(\mathrm{A})$ and $(\mathrm{B})$, require that "always available sites", $(C)$, be accounted for in $\Omega^{(B)}$. Cases III and IV, (D) and (E), require that "extra co-adsorption sites", $(F)$, be accounted for in $\Omega^{(B)}$.

\section{CASE 0: Free Translator}

To evaluate $\Omega$ for a free translator, we look specifically at the translational contribution to the free energy and disregard any rotational or vibrational degrees of freedom that may be present since they do not often contain a significant coverage dependence. For an adsorbate bound to site $\mathrm{X}$ acting as a 2D gas/free translator, its free energy is, after application of Sterling's approximation,

$$
\mathrm{F}_{\mathrm{FT}}^{(\mathrm{X})}=\mathrm{N}^{(\mathrm{X})}\left(\mathrm{V}^{(\mathrm{X})}-\mathrm{k}_{\mathrm{B}} \mathrm{T} \ln \left[\frac{\mathrm{a}_{\mathrm{u} . \mathrm{c} .}}{\lambda_{\text {th }}^{2}} \frac{\mathrm{e}}{\theta^{(\mathrm{X})}}\right]\right)
$$

where $\mathrm{F}_{\mathrm{FT}}^{(\mathrm{X})}$ and $\mathrm{V}^{(\mathrm{X})}$ are the free energy and binding energy, respectively, of an adsorbate occupying an $\mathrm{X}$ site; $\lambda_{\mathrm{th}}$ is the thermal wavelength, given by $\lambda_{\mathrm{th}}=\frac{\mathrm{h}}{\sqrt{2 \pi \mathrm{mk}_{\mathrm{B}} \mathrm{T}}}$ where $\mathrm{m}$ is the mass of the adsorbate; and $e$ is Euler's constant, i.e., $\exp (1)$. Differentiation with respect to $\mathrm{N}^{(\mathrm{X})}$ produces the $2 \mathrm{D}$ free translator chemical potential 


$$
\mu_{\mathrm{FT}}^{(\mathrm{X})}=\mathrm{V}^{(\mathrm{X})}-\mathrm{k}_{\mathrm{B}} \mathrm{T} \ln \left[\frac{\mathrm{a}_{\text {u.c. }}}{\lambda_{\text {th }}^{2}}\right]+\mathrm{k}_{\mathrm{B}} \mathrm{T} \ln \left[\theta^{(\mathrm{X})}\right]
$$

in which the second term in square brackets can be identified as the molecular partition function, $\mathrm{q}^{(\mathrm{X})}$, for a single adsorbate acting as a free translator confined to the unit cell. It is the only thermal contribution to the free energy (aside from the Boltzmann-derived linear dependence on $\mathrm{T}$ ). Thus, we can conclude that the other terms in square brackets in Equation $22 \mathrm{a}$ are $\Omega^{(\mathrm{X})}$, giving:

$$
\Omega^{(\mathrm{X})}=\left(\frac{e}{\theta^{(\mathrm{X})}}\right)^{\mathrm{N}^{(\mathrm{X})}}
$$

Note that when Equation 22b is inserted into Equation 1a, we arrive at the usual, phenomenological form of rates typically assumed in MKMs based on the law of mass action (the familiar Polanyi-Wigner expression), with chemical activity equivalent to a linear dependence on coverage (implying an activity coefficient of unity), namely

$$
r_{X \rightarrow Y}=\frac{k_{B} T}{h}\left(\frac{q_{T S}^{\ddagger}}{q^{(X)}}\right)\left(e^{\beta V^{(X)}}\right) \theta^{(X)}
$$

where $\mathrm{q}_{\mathrm{TS}}^{\ddagger}=\left(\frac{\mathrm{q}^{\ddagger}}{\mathrm{q}_{\mathrm{r.c} . \mathrm{c}}^{\ddagger}}\right)$ is the partition function of the transition state perpendicular to the reaction coordinate. It is not difficult to show that the typical $2^{\text {nd }}$ order rate law for a bimolecular reaction is the result of treating both reacting molecules as ideal $2 \mathrm{D}$ gases. For the example of $2 \mathrm{H}^{*} \rightarrow \mathrm{H}_{2}, \mu_{\mathrm{H}-\mathrm{H}}^{(\mathrm{X})}=$ $2 \mu_{\mathrm{H}^{*}}^{(\mathrm{X})}=2 \mu_{\mathrm{FT}}^{(\mathrm{X})}$, and that produces

$$
r_{X \rightarrow Y}=\frac{k_{B} T}{h}\left(\frac{q_{H-H}^{\ddagger}}{\left[q_{H^{*}}^{(X)}\right]^{2}}\right)\left(e^{\beta\left[2 v_{\mathrm{H}^{*}}^{(\mathrm{X})}\right]}\right)\left[\theta_{\mathrm{H}^{*}}^{(\mathrm{X})}\right]^{2}
$$

where subscript $\mathrm{H}^{*}$ indicates that the quantity is that of a single isolated hydrogen atom. Therefore, when we use rates of the form shown in Equations 22d and 22e in a MKM, we are tacitly assuming adsorbates behave as non-interacting 2D free translators, i.e., as an ideal gas. Such an assumption should be considered carefully as it is generally incompatible with the presence of a surface and diffusion barriers. See Section S3 of the SI for comparison of these to an ideal lattice gas formulation along with further discussion related to the physicality of rates of the form shown in Equation $22 \mathrm{~d}$.

\section{CASE I: Mutually Exclusive Co-adsorption}

Table 1. Case I-IV Example Site Blocking Schemes reflected in Figure 4.

\begin{tabular}{|c|c|c|c|}
\hline $\begin{array}{c}\text { Occupation } \\
\text { of } \mathrm{a}(\mathrm{n}) \ldots\end{array}$ & $\begin{array}{c}\text { Blocks } \\
\text { A sites }\end{array}$ & $\begin{array}{c}\text { Blocks } \\
\text { B sites }\end{array}$ & $\begin{array}{c}\text { Blocks } \\
\text { C sites }\end{array}$ \\
\hline A site & 1 & 4 & 4 \\
\hline B site & 1 & 4 & 2 \\
\hline C site & 2 & 4 & 5 \\
\hline & \multicolumn{3}{|c|}{ Case I II } \\
\hline A site & 1 & 0 & 0 \\
\hline B site & 0 & 4 & 0 \\
\hline C site & 0 & 0 & 5 \\
\hline & \multicolumn{3}{|c|}{ Case III } \\
\hline A site & 1 & 4 & 4 \\
\hline B site & 1 & 1 & 2 \\
\hline C site & 2 & 4 & 1 \\
\hline & 1 & Case IV \\
\hline A site & 0 & 0 & 0 \\
\hline B site & 0 & 0 & 1 \\
\hline C site & \multicolumn{3}{|c|}{} \\
\hline
\end{tabular}


In this first of the more realistic cases, co-adsorbates block a pre-defined number of other sites beyond the bound site. Examples of this include the adsorption of small, strongly bound molecules, e.g., $\mathrm{CO}$ and $\mathrm{NO}$, onto metal surfaces, where adsorbates typically block all sites within at least the first nearest neighbor radius, if not also within the second nearest neighbor radius. An example of this is illustrated in Figure 4A, where we have defined the number of configurations adsorbates can take on all B sites $\left(\Omega^{(\mathrm{B})}\right)$ within the figure as a concrete example. Table 1 breaks down the exact site blocking produced by occupation of each of the three site types according to this example, along with the other cases presented here (which can be found in Section S3 of the SI).

We will now construct $\Omega^{(\mathrm{A})}, \Omega^{(\mathrm{B})}$, and $\Omega^{(\mathrm{C})}$, the terms representing the number of configurations adsorbates can take when occupying $A, B$, and $C$ sites, respectively. This is a counting problem, and so we can take advantage of combinatorics to define these terms. In particular, we must find the number of combinations, given by the binomial coefficient, $\left(\begin{array}{l}n \\ k\end{array}\right)$, that $\mathrm{N}^{(\mathrm{X})}$ adsorbates occupying $\mathrm{X}$ sites can take on a surface with $\mathrm{N}_{\text {u.c. }}$ unit cells when $\mathrm{N}^{(\mathrm{Y})}$ and $\mathrm{N}^{(\mathrm{Z})}$ adsorbates already occupy a certain fixed number of $Y$ and $Z$ sites, respectively. In a typical lattice gas formulation, one would simply define the upper index of the binomial coefficient as the number of total sites and the lower index as the number of adsorbates. With sites blocked, the upper index must have the blocked sites subtracted. If we take these considerations into account, the following expression are produced:

$$
\begin{gathered}
\Omega^{(A)}=\left(\begin{array}{c}
N_{\text {u.c. }}-N^{(B)}-2 N^{(C)} \\
N^{(A)}
\end{array}\right)[1]^{\left[N^{(A)}\right]} \\
\Omega^{(B)}=\left(\begin{array}{c}
N_{\text {u.c. }}-N^{(A)}-N^{(C)} \\
N^{(B)}
\end{array}\right)[4]^{\left[N^{(B)}\right]} \\
\Omega^{(C)}=\left(\begin{array}{c}
\frac{2}{5} N_{\text {u.c. }}-\frac{4}{5} N^{(A)}-\frac{2}{5} N^{(B)} \\
N^{(C)}
\end{array}\right)[5]^{\left[N^{(C)}\right]}
\end{gathered}
$$

where we see that the coefficient on the first term in the upper index (e.g. the " $\frac{2,3}{5}$ in $\frac{2}{5} \mathrm{~N}_{\text {u.c. }}$ of Equation 23c) of each binomial coefficient represents the maximum coverage up to which adsorbates can occupy only $\mathrm{X}$ sites. This term arises from the total number of unit cells that an adsorbate effectively occupies. In the case of $A$ and $B$ sites, an adsorbate occupies the equivalent of one unit cell, but in the case of $\mathrm{C}$ sites, adsorbates occupy 2.5 unit cells. We can consider this fractional number of unit cells as an effective footprint for that site. The remaining sites are then assessed against this maximum coverage, i.e., by determining the fractional amount of these 'footprints' that are blocked by occupation of the other site types. In the case of site $\mathrm{C}$, whose own footprint has $5 \mathrm{C}$ sites in it, an occupied $\mathrm{A}$ site blocks 4 of these $C$ sites from mutual occupation. Thus, $4 / 5$ of the $C$ site footprint is blocked per adsorbate occupying $A$ sites. Likewise, occupying a $B$ site blocks $2 / 5$ of the $C$ site footprint. The upper index is modified to reflect this blocking scheme as seen in Equation 23c, and the same arguments/analyses apply to the other two sites, giving Equations 23a and 23b.

The final terms in square brackets in Equations $23 a-23 c$ account for the freedom of movement each adsorbate has within its own footprint. The binomial coefficients effectively count the number of configurations that $\mathrm{N}^{(\mathrm{X})}$ adsorbates can take amongst all available X-site footprints as opposed to sites. However, since an adsorbate blocks its own sites, these sites are always available for occupation by that adsorbate, see Figure 4C. Since movement to one of these sites simply produces an identical footprint with exactly the same number of sites/footprints blocked, the binomial coefficient is unchanged, and these "always available" sites must therefore be counted separately. In the case of A sites, there is only $1 \mathrm{~A}$ site per footprint, and so there are no new configurations to account for. However, both $B$ and $C$ sites have extra sites that are always available within their own footprints: 4 sites per occupied $B$ site and 5 sites per occupied $C$ site. As a result, the number of new configurations is given (per adsorbate occupying an $\mathrm{X}$ site) by the product of always available sites within each footprint: $\left[b^{(X)}\right]^{N^{(X)}}$, where $b^{(X)}$ is the total number of $X$ sites blocked by occupation of an $X$ site.

Application of Sterling's Approximation to the binomial coefficients in Equations $23 a-23 c$ gives 


$$
\begin{gathered}
\Omega^{(\mathrm{A})}=\left[\frac{\left(1-\theta^{(\mathrm{B})}-2 \theta^{(\mathrm{C})}\right)^{\left(1-\theta^{(\mathrm{B})}-2 \theta^{(\mathrm{C})}\right)}}{\left(\theta^{(\mathrm{A})}\right)^{\left(\theta^{(\mathrm{A})}\right)}\left(1-\theta-\theta^{(\mathrm{C})}\right)^{\left(1-\theta-\theta^{(\mathrm{C})}\right)}}\right]^{\mathrm{N}_{\text {u.c. }}} \\
\Omega^{(\mathrm{B})}=\left[\frac{\left(1-\theta^{(\mathrm{A})}-\theta^{(\mathrm{C})}\right)^{\left(1-\theta^{(\mathrm{B})}-\theta^{(\mathrm{C})}\right)}}{\left(\theta^{(\mathrm{B})}\right)^{\left(\theta^{(\mathrm{B})}\right)}(1-\theta)^{(1-\theta)}} 4^{\left[\theta^{(\mathrm{B})}\right]}\right]^{\mathrm{N}_{\text {u.c. }}} \\
\Omega^{(\mathrm{C})}=\left[\left(\frac{\left(1-2 \theta^{(\mathrm{A})}-\theta^{(\mathrm{B})}\right)^{\left(1-2 \theta^{(\mathrm{A})}-\theta^{(\mathrm{B})}\right)}}{\left.\left(\left(\frac{5}{2} \theta^{(\mathrm{C})}\right)^{\left(\frac{5}{2} \theta^{(\mathrm{C})}\right]}\right)^{(\mathrm{C})}\right)}\right]^{\frac{2}{5}}\right]^{\mathrm{N}_{\text {u.c. }}}\left(1-2 \theta^{(\mathrm{A})}-\theta^{(\mathrm{B})}-\frac{5}{2} \theta^{(\mathrm{C})}\right)^{\left(1-2 \theta^{(\mathrm{A})}-\theta^{(\mathrm{B})}-\frac{5}{2} \theta^{(\mathrm{C})}\right)}
\end{gathered}
$$

where the terms in large square brackets represent $\omega^{(\mathrm{A})}, \omega^{(\mathrm{B})}$, and $\omega^{(\mathrm{C})}$, respectively; which can be inserted into Equation 6 to fully define the free energy and entropy of the entire surface; or differentiated with respect to each site coverage and inserted into Equation 13 to yield the chemical potential and chemical activity of each occupied site.

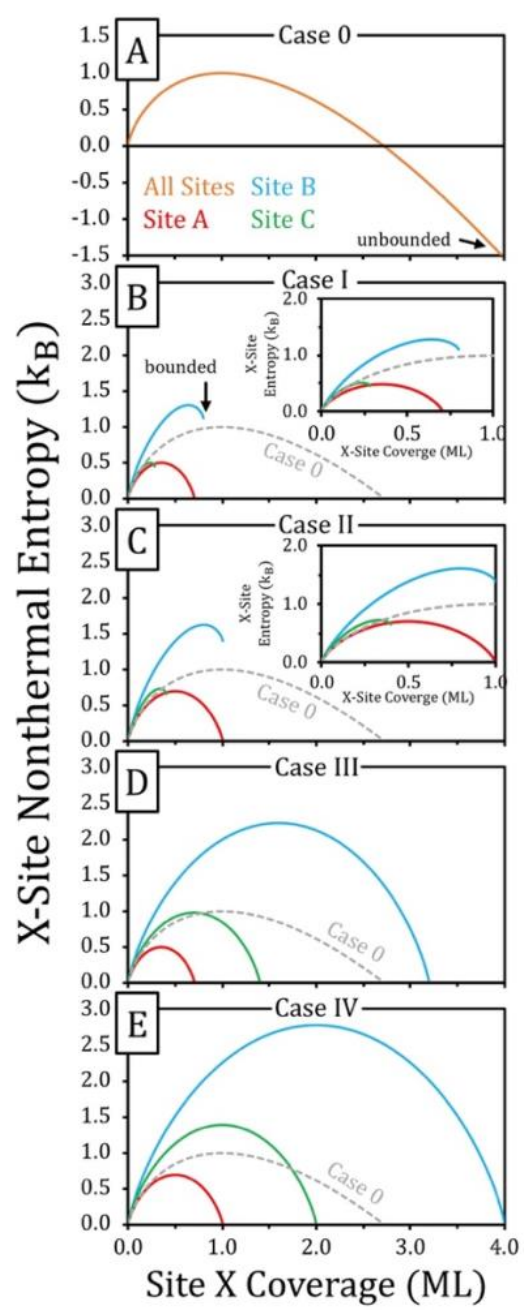

Figure 5. Resulting nonthermal entropy $\left(\mathrm{k}_{\mathrm{B}} \ln \left(\omega^{(\mathrm{X})}\right)\right.$, specifically) for each site type, $\mathrm{A}$ (red curves), $\mathrm{B}$ (blue curves), and C (green curves) for co-adsorption site-blocking (A) Case 0, (B) Case I, (C) Case II, (D) Case III, and (E) Case IV in units of $\mathrm{k}_{\mathrm{B}}$. Here, both site coverages for non-variable sites are fixed at $0.1 \mathrm{ML}$. Because of the small domain and range of the entropy expressions under Case I and II, a zoomed in view is provided inset each panel. 
Derivation of Cases II - IV is similar to that of Case I and shown in Section S3 of the SI. The resultant nonthermal entropy (second term in Equation 5b) for each case-including that of a 2D free translator, Case 0-are compared in Figure 5. The thermal entropy contribution from each site that comes from the site partition functions (first term in Equation $\mathbf{5 b}$ ) is not included here because it is system dependent. Additionally, it is from the nonthermal entropy that the chemical activity of each site is derived, while the thermal entropy is typically subsumed into the preexponential factor. Because the extent of the surface is explicitly accounted for, the allowed coverages are bounded on both ends by zero and the upper index of the associated combinatoric. Case 0, on the other hand, is only bounded at zero and is otherwise unbounded-toward negative infinity-as coverage increases, which is an aphysical prediction for a finite surface or volume. This unbounded entropy occurs because 2D free translators behave as an ideal gas having access to the entire surface regardless of how many adsorbates are present: there is no limit to the number of adsorbates that can fit into the system. The entropy trends toward negative infinity because 2D free translators are indistinguishable, and the number of degenerate energy states increases exponentially (more accurately: factorially) with the number of indistinguishable adsorbates, causing an unbounded decline in the number of unique configurations responsible for the adsorbates' entropies according to the ideal gas model. Additionally, while Case 0 has a maximum in its entropy contribution at an intermediate value (like for Cases IIV), its maximum is at $1 \mathrm{ML}$, which would often correspond to saturation coverage in many systems. In fact, because there is no way to entropically set an upper bound in Case 0 , this result cannot change regardless of what the system is or the active site it represents. If we artificially bound the coverage to $1 \mathrm{ML}$, Case 0 acts qualitatively like the other, more physical cases, which possibly explains why this approximation has been used as successfully as it has in the literature.

\section{Results and Discussion}

\subsection{Demonstrated Thermodynamic Self-Consistency}

We will now construct example MF-MKMs for a simple adsorption/desorption process of a monatomic gas to/from a surface with three site types: $A, B$, and C. Each MF-MKM system will be exposed to a single reservoir chemical potential with the only adsorption/desorption occ(so that steady state total coverage and site fractions can be compared against their equilibrium values predicted from the system's free energy. This will allow us to assess the error in models that might stem from a lack of thermodynamic self-consistency, while showing the ease of congruence when one uses the expressions derived here. We will look at two scenarios. The first scenario is one in which the adsorbates are treated as non-interacting 2D translators, where a model consistent with the diffusion and adsorption/desorption rates derived here (Equations 11 and 18) can be compared against a version where individual desorption rates are used instead (as opposed to a single desorption rate as defined in Equation 18a). This is the only scenario that permits a sum of individual desorption rates to exhibit thermodynamic self-consistency. The second scenario is one in which adsorbates are treated more realistically, with one model accounting for realistic site blocking to produce coverage dependence (i.e., chemical activity) and another assuming the chemical activity of each site is equivalent to its coverage as the law of mass action would typically suggest.

By defining the free energy of the system explicitly (via Equation 5), we can determine the equilibrium coverage and distribution amongst site types by minimizing the system's free energy and comparing this to what the microkinetic models predict. The total free energy of the entire system is defined as

$$
\mathrm{F}_{\text {total }}=\mathrm{F}_{\text {surf }}+\mathrm{F}_{\text {res }}
$$

where $F_{\text {surf }}$ is the free energy of the surface + adsorbates, and $F_{\text {res }}$ is the free energy of the gas reservoir. Without lateral/molecular interaction, adsorption will be Langmuirian, resulting only in saturation coverage. So a 2-body lateral interaction term will be applied equally to all sites to provide some variation in equilibrium coverages, giving

$$
\begin{aligned}
\mathrm{F}_{\text {surf }}=\mathrm{N}_{\text {u.c. }}\left[\mathrm{V}^{(\mathrm{A})} \theta^{(\mathrm{A})}+\mathrm{V}^{(\mathrm{B})} \theta^{(\mathrm{B})}+\mathrm{V}^{(\mathrm{C})} \theta^{(\mathrm{C})}+\frac{\mathrm{c}}{2} \mathrm{~V}_{\text {int }} \theta^{2}-\theta^{(\mathrm{A})} \mathrm{k}_{\mathrm{B}} \mathrm{T} \ln \left(\mathrm{q}^{(\mathrm{A})}\right)\right. \\
\left.-\theta^{(\mathrm{B})} \mathrm{k}_{\mathrm{B}} \mathrm{T} \ln \left(\mathrm{q}^{(\mathrm{B})}\right)-\theta^{(\mathrm{C})} \mathrm{k}_{\mathrm{B}} \mathrm{T} \ln \left(\mathrm{q}^{(\mathrm{C})}\right)-\mathrm{k}_{\mathrm{b}} \mathrm{T} \ln \left(\omega^{(\mathrm{A})} \omega^{(\mathrm{B})} \omega^{(\mathrm{C})}\right)\right]
\end{aligned}
$$


where $\mathrm{V}^{(\mathrm{A})}, \mathrm{V}^{(\mathrm{B})}$, and $\mathrm{V}^{(\mathrm{C})}$ are the "zero coverage" adsorption energies of the adsorbate occupying the $A, B$, and $C$ sites, respectively; $c$ is the coordination number of the surface; and $V_{\text {int }}$ is the lateral interaction energy (i.e., $V_{\text {lat }}=\frac{c}{2} V_{\text {int }} \theta^{2}$ ). We could define $F_{\text {res }}$ as $N \mu_{\text {res }}$, but to have the adsorption/desorption rate expressions resemble those typically encountered, we choose to define the free energy explicitly as that of an ideal (3D) gas:

$$
\mathrm{F}_{\text {res }}=\mathrm{F}_{\text {res }}^{0}+\mathrm{k}_{\mathrm{B}} \mathrm{TN} \ln \left(\frac{\mathrm{k}_{\mathrm{B}} \mathrm{T}}{\lambda_{\text {th }}^{3} \mathrm{P}_{\text {res }}}\right)
$$

where $\mathrm{F}_{\text {res }}^{0}$ is the free energy of a molecule in the reservoir prior to adsorption, which is treated as the reference energy for the entire system and is thus equal to 0 here; and $\mathrm{P}_{\text {res }}$ is the (partial) pressure of the molecule in the reservoir. As written, Equation 25 is extensive, dependent on the size of the system, $\mathrm{N}_{\text {u.c. }}$; but chemical potentials and the minimization of $\mathrm{F}_{\text {total }}$ are not. We will thus divide by $\mathrm{N}_{\text {u.c. }}$ to produce the analogous intensive variable, which will be the value reported when free energies are shown. Note that holding pressure constant in Equation 25c makes all free energies Gibbs free energies.

\section{SCENARIO 1}

To make these models (which we will denote as model $\alpha$ and $\beta$ ) as similar to those usually used in microkinetic modeling as possible, the adsorbates are treated as ideal 2D translators in the first scenario. This means in any given configuration, an adsorbate occupying site $A$, site $B$, or site $C$ will experience the same unhindered translational movement. This movement will be restricted only to sites of the same type, however, to inject some physicality back into the model. The molecular partition functions for all other degrees of freedom will be set to unity for simplicity. Thus,

$$
\begin{aligned}
& q^{(A)}=\frac{a_{S}^{(A)}}{\lambda_{t h}^{2}} \\
& q^{(B)}=\frac{a_{S}^{(B)}}{\lambda_{t h}^{2}} \\
& q^{(C)}=\frac{a_{S}^{(C)}}{\lambda_{t h}^{2}}
\end{aligned}
$$

where $\mathrm{a}_{\mathrm{S}}^{(\mathrm{A})}, \mathrm{a}_{\mathrm{S}}^{(\mathrm{B})}$, and $\mathrm{a}_{\mathrm{S}}^{(\mathrm{C})}$ are the areas of site $\mathrm{A}, \mathrm{B}$, and $\mathrm{C}$, respectively. For our purposes here, we will define as the fraction of the unit cell covered in those sites, i.e., $a_{S}^{(X)}=\frac{n^{(X)}}{n^{(A)}+n^{(B)}+n^{(C)}} a_{u . c .}$. This then allows the additional entropy gained from movement between unit cells to be captured in the $\omega^{(\mathrm{A})}$ an $\omega^{(B)}$ terms. From Equation 22c, these are

$$
\begin{aligned}
& \omega^{(\mathrm{A})}=\left(\frac{e}{\theta^{(\mathrm{A})}}\right)^{\mathrm{N}^{(\mathrm{A})}} \\
& \omega^{(\mathrm{B})}=\left(\frac{e}{\theta^{(\mathrm{B})}}\right)^{\mathrm{N}^{(\mathrm{B})}} \\
& \omega^{(\mathrm{C})}=\left(\frac{e}{\theta^{(\mathrm{C})}}\right)^{\mathrm{N}^{(\mathrm{C})}}
\end{aligned}
$$

After inserting Equations 26a - 26e into Equation 25, the chemical potentials of an adsorbate occupying site $A$ and site $B$ are found by differentiating $F_{\text {surf }}$ with respect to $N^{(A)}, N^{(B)}$, and $N^{(C)}$ (shown in Equation 12) to give

$$
\begin{aligned}
& \mu^{(\mathrm{A})}=\mathrm{V}^{(\mathrm{A})}+\mathrm{cV}_{\text {int }} \theta-\mathrm{k}_{\mathrm{B}} \mathrm{T} \ln \left[\frac{\mathrm{a}_{\mathrm{S}}^{(\mathrm{A})}}{\lambda_{\text {th }}^{2}}\right]+\mathrm{k}_{\mathrm{B}} \mathrm{T} \ln \left[\theta^{(\mathrm{A})}\right] \\
& \mu^{(\mathrm{B})}=\mathrm{V}^{(\mathrm{B})}+\mathrm{cV}_{\text {int }} \theta-\mathrm{k}_{\mathrm{B}} \mathrm{T} \ln \left[\frac{\mathrm{a}_{\mathrm{S}}^{(\mathrm{B})}}{\lambda_{\text {th }}^{2}}\right]+\mathrm{k}_{\mathrm{B}} \mathrm{T} \ln \left[\theta^{(\mathrm{B})}\right]
\end{aligned}
$$




$$
\mu^{(\mathrm{C})}=\mathrm{V}^{(\mathrm{C})}+\mathrm{cV}_{\text {int }} \theta-\mathrm{k}_{\mathrm{B}} \mathrm{T} \ln \left[\frac{\mathrm{a}_{\mathrm{S}}^{(\mathrm{C})}}{\lambda_{\mathrm{th}}^{2}}\right]+\mathrm{k}_{\mathrm{B}} \mathrm{T} \ln \left[\theta^{(\mathrm{C})}\right]
$$

The chemical potential of the reservoir is found by differentiating $\mathrm{F}_{\text {res }}$ with respect to $\mathrm{N}$, but because $\mathrm{N}$ is the number of adsorbates removed from the reservoir, we must also reverse the sign to yield

$$
\mu_{\text {res }}=-\mathrm{k}_{\mathrm{B}} \mathrm{T} \ln \left(\frac{\mathrm{k}_{\mathrm{B}} \mathrm{T}}{\lambda_{\text {th }}^{3} \mathrm{P}_{\mathrm{res}}}\right)
$$

Since we are interested in comparing equilibrium values and general trends, we will define the chemical potential of any activated complex based only on the partition function of the degree of freedom corresponding to the reaction coordinate. Thus,

$$
\mu_{\mathrm{AB}}^{\ddagger}=-\mathrm{k}_{\mathrm{B}} \mathrm{T} \ln \left[\mathrm{q}_{\text {r.c. }}^{\ddagger}\right]
$$

which, due to the presence of $q_{\text {r.c. }}^{\ddagger}$ in the denominator of Equation 1, is equivalent to assuming the transition state has zero degrees of freedom perpendicular to the reaction coordinate and is otherwise at the energetic zero of the system. The transition state for adsorption/desorption is, as usually done, assumed to belong to an ideal gas molecule moving along a reaction coordinate of arbitrary length, $\delta$, confined to a volume equal to this length multiplied by the area of a unit cell. This gives that

$$
\mu_{\text {surf-res }}^{\ddagger}=-\mathrm{k}_{\mathrm{B}} \mathrm{T} \ln \left(\frac{\delta \mathrm{a}_{\text {u.c. }}}{\lambda_{\text {th }}^{3}}\right)
$$

or, for desorption from a single site type

$$
\mu_{\mathrm{X}-\mathrm{res}}^{\ddagger}=-\mathrm{k}_{\mathrm{B}} \mathrm{T} \ln \left(\frac{\delta \mathrm{a}_{\mathrm{S}}^{(\mathrm{X})}}{\lambda_{\mathrm{th}}^{3}}\right)
$$

which will be used in Model $\beta$. Note that the adsorption/desorption transition state is also at the energetic zero of the system since we assume adsorption to be unactivated. This means that in this scenario, the activation barriers for diffusion and desorption will be similar in magnitude.

For adsorption/desorption, $\mathrm{q}_{\text {r.c. }}^{\ddagger}$ must be defined explicitly. It is the partition function at the transition state projected along the reaction coordinate, which in this case, is simply an ideal 1D gas confined to $\delta$, namely

$$
\mathrm{q}_{\text {r.c. }}^{\ddagger}=\frac{\delta}{\lambda_{\text {th }}}
$$

Substituting Equations 26 - 28 into Equations 12 and 19

produces the rate expressions to be used in our microkinetic model:

\section{MODEL $\alpha$}

$$
\begin{aligned}
& r_{A \rightarrow B}=r_{A \rightarrow C}=\frac{k_{B} T}{h} e^{\beta\left[\mu^{(A)}\right]}=\frac{k_{B} T}{h}\left[n^{(A)}+n^{(B)}+n^{(C)}\right]\left[\frac{\lambda_{t h}^{2}}{a_{\text {u.c. }}}\right]\left[\frac{\theta^{(A)}}{n^{(A)}}\right] e^{\beta\left[V^{(A)}+c V_{\text {int }} \theta\right]} \\
& r_{B \rightarrow A}=r_{B \rightarrow C}=\frac{k_{B} T}{h} e^{\beta\left[\mu^{(B)}\right]}=\frac{k_{B} T}{h}\left[n^{(A)}+n^{(B)}+n^{(C)}\right]\left[\frac{\lambda_{t h}^{2}}{a_{\text {u.c. }}}\right]\left[\frac{\theta^{(B)}}{n^{(B)}}\right] e^{\beta\left[v^{(B)}+c V_{i n t} \theta\right]} \\
& r_{C \rightarrow A}=r_{C \rightarrow B}=\frac{k_{B} T}{h} e^{\beta\left[\mu^{(C)}\right]}=\frac{k_{B} T}{h}\left[n^{(A)}+n^{(B)}+n^{(C)}\right]\left[\frac{\lambda_{\text {th }}^{2}}{a_{\text {u.c. }}}\right]\left[\frac{\theta^{(C)}}{n^{(C)}}\right] e^{\beta\left[v^{(C)}+c V_{\text {int }} \theta\right]} \\
& r_{\text {surf } \rightarrow \text { res }}=S_{0}(1-\theta) \frac{\mathrm{k}_{\mathrm{B}} \mathrm{T}}{\mathrm{h}} \frac{\mathrm{a}_{\text {u.c. }}}{\lambda_{\text {th }}^{2}} \mathrm{e}^{\beta\left[\mathrm{y}^{(\mathrm{A})} \mu^{(\mathrm{A})}+\mathrm{y}^{(\mathrm{B})} \mu^{(\mathrm{B})}+\mathrm{y}^{(\mathrm{C})} \mu^{(\mathrm{C})}\right]} \\
& =S_{0}(1-\theta) \frac{k_{B} T}{h}\left[n^{(A)}+n^{(B)}+n^{(C)}\right]\left[\frac{\theta^{(A)}}{n^{(A)}}\right]^{y^{(A)}}\left[\frac{\theta^{(B)}}{n^{(B)}}\right]^{y^{(B)}}\left[\frac{\theta^{(C)}}{n^{(C)}}\right]^{y^{(C)}} e^{\beta\left[y^{(A)}\left(V^{(A)}+c v_{\text {int }} \theta\right)+y^{(B)}\left(v^{(B)}+c V_{\text {int }} \theta\right)+y^{(C)}\left(v^{(C)}+c V_{\text {int }} \theta\right)\right]} \\
& r_{\text {res } \rightarrow \text { surf }}=S_{0}(1-\theta) \frac{P_{\text {res }} a_{\text {u.c. }}}{\sqrt{2 \pi \mathrm{mk}_{\mathrm{B}} \mathrm{T}}}
\end{aligned}
$$

where one can now note the importance of the number of site types per supercell, $\mathrm{n}^{(\mathrm{X})}$, as they appear explicitly in each expression except for the adsorption rate (Equation 29e). Note that we have appended a direct sticking coefficients, $S_{0}(1-\theta)$, which accounts for the (in)efficiency of energy 
transfer to and from the surface when an adsorbate impinges on it. To ensure detail balance, the sticking coefficient must also affect the desorption rate. Its presence does not affect thermodynamic self-consistency; it is a purely kinetic term. Note however that this is the simplest sticking scheme as it does not subsume the effect of lateral interactions between the adspecies.

We will compare this to a more common version of the rate expressions where individual adsorption/desorption rates are provided for individual site types. The diffusion rates will be unchanged. This gives:

\section{MODEL $\beta$}

$$
\begin{aligned}
& r_{A \rightarrow \text { res }}=S_{0}\left(1-\theta^{(A)}\right) \frac{k_{B} T}{h} \frac{1}{q_{\text {r.c. }}^{\ddagger}} e^{-\beta\left[\mu_{A-r e s}^{\ddagger}-\mu^{(A)}\right]}=S_{0}\left(1-\theta^{(A)}\right) \frac{k_{B} T}{h} \frac{a_{S}^{(A)}}{\lambda_{\text {th }}^{2}} e^{\beta\left[V^{(A)}+c V_{\text {lat }} \theta\right]} \theta^{(A)} \\
& r_{\text {res } \rightarrow A}=S_{0}\left(1-\theta^{(A)}\right) \frac{P_{r e s} a_{S}^{(A)}}{\sqrt{2 \pi \mathrm{mk}_{B} T}} \\
& r_{B \rightarrow \text { res }}=S_{0}\left(1-\theta^{(B)}\right) \frac{k_{B} T}{h} \frac{1}{q_{r . c .}^{\ddagger}} e^{-\beta\left[\mu_{B-r e s}^{\ddagger}-\mu^{(B)}\right]}=S_{0}\left(1-\theta^{(B)}\right) \frac{k_{B} T}{h} \frac{a_{S}^{(B)}}{\lambda_{t h}^{2}} e^{\beta\left[v^{(B)}+c V_{\text {lat } \theta} \theta\right.} \theta^{(B)} \\
& r_{\text {res } \rightarrow B}=S_{0}\left(1-\theta^{(B)}\right) \frac{P_{r e s} a_{S}^{(B)}}{\sqrt{2 \pi \mathrm{k}_{B} \mathrm{~T}}} \\
& \mathrm{r}_{\mathrm{C} \rightarrow \mathrm{res}}=\mathrm{S}_{0}\left(1-\theta^{(\mathrm{C})}\right) \frac{\mathrm{k}_{\mathrm{B}} \mathrm{T}}{\mathrm{h}} \frac{1}{\mathrm{q}_{\mathrm{r} . \mathrm{c} .}^{\ddagger}} \mathrm{e}^{-\beta\left[\mu_{\mathrm{C}-\mathrm{res}}^{\ddagger}-\mu^{(\mathrm{C})}\right]}=\mathrm{S}_{0}\left(1-\theta^{(\mathrm{C})}\right) \frac{\mathrm{k}_{\mathrm{B}} \mathrm{T}}{\mathrm{h}} \frac{\mathrm{a}_{\mathrm{S}}^{(\mathrm{C})}}{\lambda_{\text {th }}^{2}} \mathrm{e}^{\beta\left[\mathrm{v}^{(\mathrm{C})}+c \mathrm{~V}_{\text {lat }} \theta\right]} \theta^{(\mathrm{C})} \\
& r_{\text {res } \rightarrow C}=S_{0}\left(1-\theta^{(C)}\right) \frac{P_{r e s} a_{S}^{(C)}}{\sqrt{2 \pi k_{B} T}}
\end{aligned}
$$

where supposed sticking coefficients have again been added according to the rate being defined.

We can now show how Model $\beta$ is setup in the only way that satisfies thermodynamic selfconsistency using individual desorption rates, which can be generalized as:

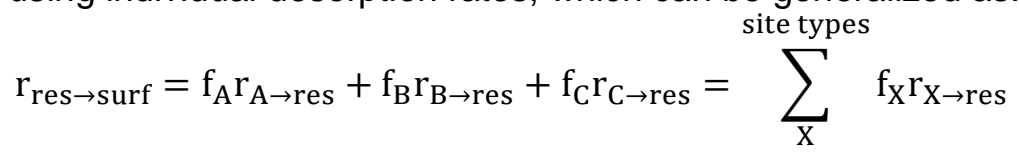

where

$$
\sum_{\mathrm{X}}^{\text {site types }} \mathrm{f}_{\mathrm{X}}=1
$$

and is only valid if and only if

$$
\mathrm{r}_{\mathrm{A} \rightarrow \mathrm{res}}=\mathrm{r}_{\mathrm{B} \rightarrow \mathrm{res}}=\mathrm{r}_{\mathrm{C} \rightarrow \mathrm{res}}
$$

which is the case at equilibrium where $\mu^{(\mathrm{A})}=\mu^{(\mathrm{B})}=\mu^{(\mathrm{C})}$ but is otherwise violated when the system is outside equilibrium conditions. Thus, Model $\beta$ is thermodynamic self-consistent because (1) diffusion rates are properly defined according to Equation 12 so that Equation $31 \mathrm{c}$ is satisfied at equilibrium, and (2) because we have defined transition state chemical potentials for each desorption/adsorption rate (Equation 28b and 28c) that are proportional to the fraction of the surface covered in $X$ sites, which satisfies Equation 31b so that

$$
\sum_{X}^{\text {site types }} q_{X \rightarrow r e s}^{\ddagger}=q_{\text {res } \rightarrow \text { surf }}^{\ddagger}
$$

where $\mathrm{q}_{\mathrm{X} \rightarrow \mathrm{res}}^{\ddagger}=\mathrm{e}^{-\beta \mu_{\mathrm{X} \rightarrow \mathrm{res}}^{\ddagger}}=\mathrm{f}_{\mathrm{X}}$ and $\mathrm{q}_{\mathrm{res} \rightarrow \text { surf }}^{\ddagger}=\mathrm{e}^{-\beta \mu_{\mathrm{res} \rightarrow \text { surf }}^{\ddagger}}=\sum_{\mathrm{X}}^{\text {site types }} \mathrm{q}_{\mathrm{res} \rightarrow \mathrm{X}}^{\ddagger}$. It will become clear how the coincidental validity of Model $\beta$ nonetheless produces aphysical behavior in the kinetics.

The rate expression in Equations 29 and 30 allow us to define the system of ordinary differential equations (ODEs) that comprises each microkinetic model, which is established via mole balance around each site type and the surface/reservoir. These are 
MODEL $\alpha$

$$
\begin{gathered}
\frac{d \theta^{(\mathrm{A})}}{d t}=-\mathrm{r}_{\mathrm{A} \rightarrow \mathrm{B}}-\mathrm{r}_{\mathrm{A} \rightarrow \mathrm{C}}+\mathrm{r}_{\mathrm{B} \rightarrow \mathrm{A}}+\mathrm{r}_{\mathrm{C} \rightarrow \mathrm{A}}+\mathrm{y}^{(\mathrm{A})}\left[\frac{d \theta}{d t}\right] \\
\frac{d \theta^{(\mathrm{B})}}{d t}=-\mathrm{r}_{\mathrm{B} \rightarrow \mathrm{A}}-\mathrm{r}_{\mathrm{B} \rightarrow \mathrm{C}}+\mathrm{r}_{\mathrm{A} \rightarrow \mathrm{B}}+\mathrm{r}_{\mathrm{C} \rightarrow \mathrm{B}}+\mathrm{y}^{(\mathrm{B})}\left[\frac{d \theta}{d t}\right] \\
\frac{d \theta^{(\mathrm{C})}}{d t}=-\mathrm{r}_{\mathrm{C} \rightarrow \mathrm{A}}-\mathrm{r}_{\mathrm{C} \rightarrow \mathrm{B}}+\mathrm{r}_{\mathrm{A} \rightarrow \mathrm{C}}+\mathrm{r}_{\mathrm{B} \rightarrow \mathrm{C}}+\mathrm{y}^{(\mathrm{C})}\left[\frac{d \theta}{d t}\right] \\
\frac{d \theta}{d t}=-\mathrm{r}_{\text {surf } \rightarrow \text { res }}+\mathrm{r}_{\text {res } \rightarrow \text { surf }}
\end{gathered}
$$

MODEL $\beta$

$$
\begin{gathered}
\frac{d \theta^{(\mathrm{A})}}{d t}=-\mathrm{r}_{\mathrm{A} \rightarrow \mathrm{B}}-\mathrm{r}_{\mathrm{A} \rightarrow \mathrm{C}}+\mathrm{r}_{\mathrm{B} \rightarrow \mathrm{A}}+\mathrm{r}_{\mathrm{C} \rightarrow \mathrm{A}}-\mathrm{r}_{\mathrm{A} \rightarrow \mathrm{res}}+\mathrm{r}_{\mathrm{res} \rightarrow \mathrm{A}} \\
\frac{d \theta^{(\mathrm{B})}}{d t}=-\mathrm{r}_{\mathrm{B} \rightarrow \mathrm{A}}-\mathrm{r}_{\mathrm{B} \rightarrow \mathrm{C}}+\mathrm{r}_{\mathrm{A} \rightarrow \mathrm{B}}+\mathrm{r}_{\mathrm{C} \rightarrow \mathrm{B}}-\mathrm{r}_{\mathrm{B} \rightarrow \mathrm{res}}+\mathrm{r}_{\mathrm{res} \rightarrow \mathrm{B}} \\
\frac{d \theta^{(\mathrm{C})}}{d t}=-\mathrm{r}_{\mathrm{C} \rightarrow \mathrm{A}}-\mathrm{r}_{\mathrm{C} \rightarrow \mathrm{B}}+\mathrm{r}_{\mathrm{A} \rightarrow \mathrm{C}}+\mathrm{r}_{\mathrm{B} \rightarrow \mathrm{C}}-\mathrm{r}_{\mathrm{C} \rightarrow \mathrm{res}}+\mathrm{r}_{\mathrm{res} \rightarrow \mathrm{C}} \\
\frac{d \theta}{d t}=-\mathrm{r}_{\mathrm{A} \rightarrow \mathrm{res}}-\mathrm{r}_{\mathrm{B} \rightarrow \mathrm{res}}-\mathrm{r}_{\mathrm{C} \rightarrow \mathrm{res}}+\mathrm{r}_{\mathrm{res} \rightarrow \mathrm{A}}+\mathrm{r}_{\mathrm{res} \rightarrow \mathrm{B}}+\mathrm{r}_{\mathrm{res} \rightarrow \mathrm{C}}
\end{gathered}
$$

It is desirable to recast these into the identified independent variables of $\theta$ and $\mathrm{y}^{(\mathrm{X})}$ :

$$
\frac{d \theta^{(\mathrm{X})}}{d t}=\theta \frac{d \mathrm{y}}{d t}+\mathrm{y}(\mathrm{X}) \frac{d \theta}{d t}
$$

Substituting Equation 34 into Equations $32-33$ and rearranging produces

$\underline{\text { MODEL } \alpha}$

$$
\begin{aligned}
& \frac{d \mathrm{y}^{(\mathrm{A})}}{d t}=\frac{-\mathrm{r}_{\mathrm{A} \rightarrow \mathrm{B}}-\mathrm{r}_{\mathrm{A} \rightarrow \mathrm{C}}+\mathrm{r}_{\mathrm{B} \rightarrow \mathrm{A}}+\mathrm{r}_{\mathrm{C} \rightarrow \mathrm{A}}}{\theta} \\
& \frac{d \mathrm{y}^{(\mathrm{B})}}{d t}=\frac{-\mathrm{r}_{\mathrm{B} \rightarrow \mathrm{A}}-\mathrm{r}_{\mathrm{B} \rightarrow \mathrm{C}}+\mathrm{r}_{\mathrm{A} \rightarrow \mathrm{B}}+\mathrm{r}_{\mathrm{C} \rightarrow \mathrm{B}}}{\theta} \\
& \frac{d \mathrm{y}^{(\mathrm{C})}}{d t}=\frac{-\mathrm{r}_{\mathrm{C} \rightarrow \mathrm{A}}-\mathrm{r}_{\mathrm{C} \rightarrow \mathrm{B}}+\mathrm{r}_{\mathrm{A} \rightarrow \mathrm{C}}+\mathrm{r}_{\mathrm{B} \rightarrow \mathrm{C}}}{\theta} \\
& \frac{d \theta}{d t}=-\mathrm{r}_{\text {surf } \rightarrow \text { res }}+\mathrm{r}_{\text {res } \rightarrow \text { surf }}
\end{aligned}
$$

MODEL $\beta$

$$
\begin{aligned}
& \frac{d \mathrm{y}^{(\mathrm{A})}}{d t}=\frac{-\mathrm{r}_{\mathrm{A} \rightarrow \mathrm{B}}-\mathrm{r}_{\mathrm{A} \rightarrow \mathrm{C}}+\mathrm{r}_{\mathrm{B} \rightarrow \mathrm{A}}+\mathrm{r}_{\mathrm{C} \rightarrow \mathrm{A}}-\mathrm{r}_{\mathrm{A} \rightarrow \mathrm{res}}+\mathrm{r}_{\mathrm{res} \rightarrow \mathrm{A}}-\mathrm{y}^{(\mathrm{A})}\left[\frac{d \theta}{d t}\right]}{\theta} \\
& \frac{d \mathrm{y}^{(\mathrm{B})}}{d t}=\frac{-\mathrm{r}_{\mathrm{B} \rightarrow \mathrm{A}}-\mathrm{r}_{\mathrm{B} \rightarrow \mathrm{C}}+\mathrm{r}_{\mathrm{A} \rightarrow \mathrm{B}}+\mathrm{r}_{\mathrm{C} \rightarrow \mathrm{B}}-\mathrm{r}_{\mathrm{B} \rightarrow \mathrm{res}}+\mathrm{r}_{\mathrm{res} \rightarrow \mathrm{B}}-\mathrm{y}^{(\mathrm{B})}\left[\frac{d \theta}{d t}\right]}{\theta} \\
& \frac{d \mathrm{y}^{(\mathrm{C})}}{d t}=\frac{-\mathrm{r}_{\mathrm{C} \rightarrow \mathrm{A}}-\mathrm{r}_{\mathrm{C} \rightarrow \mathrm{B}}+\mathrm{r}_{\mathrm{A} \rightarrow \mathrm{C}}+\mathrm{r}_{\mathrm{B} \rightarrow \mathrm{C}}-\mathrm{r}_{\mathrm{C} \rightarrow \mathrm{res}}+\mathrm{r}_{\mathrm{res} \rightarrow \mathrm{C}}-\mathrm{y}^{(\mathrm{C})\left[\frac{d \theta}{d t}\right]}}{\theta} \\
& \frac{d \theta}{d t}=-\mathrm{r}_{\mathrm{A} \rightarrow \mathrm{res}}-\mathrm{r}_{\mathrm{B} \rightarrow \mathrm{res}}-\mathrm{r}_{\mathrm{C} \rightarrow \mathrm{res}}+\mathrm{r}_{\text {res } \rightarrow \mathrm{A}}+\mathrm{r}_{\mathrm{res} \rightarrow \mathrm{B}}+\mathrm{r}_{\mathrm{res} \rightarrow \mathrm{C}}
\end{aligned}
$$

where model $\alpha$ is well simplified (upon division by $\theta$, the ODEs in Equation 35a - 35c simplify yet further), and model $\beta$ is made much less simple, hinting at its inappropriateness. In practice, we will 
use a molar balance to define the full set of equations needed for all models (replacing one of the ODEs); but in principle, this is optional. The parameters used in Equation 25 are shown along with the initial values of each variable are shown in Table 2.

Table 2. Parameters and initial values used in MF-MKMs of Model $\alpha$ and $\beta$ for Scenario 1.

\begin{tabular}{|c|c|}
\hline Parameter & Value \\
\hline $\mathrm{T}$ & $300 \mathrm{~K}$ \\
\hline $\mathrm{P}_{\text {res }}$ & $1 \mathrm{bar}$ \\
\hline $\mathrm{m}$ & $30 \mathrm{~g} / \mathrm{mol}$ \\
\hline $\mathrm{a}_{\text {u.c. }}$ & $25 \AA^{2}$ \\
\hline $\mathrm{V}^{(\mathrm{A})}$ & $-1.00 \mathrm{eV}$ \\
\hline $\mathrm{V}^{(\mathrm{B})}$ & $-0.90 \mathrm{eV}$ \\
\hline $\mathrm{V}^{(\mathrm{C})}$ & $-0.85 \mathrm{eV}$ \\
\hline $\mathrm{n}^{(\mathrm{A})}$ & 1 \\
\hline $\mathrm{n}^{(\mathrm{B})}$ & 3 \\
\hline $\mathrm{n}^{(\mathrm{C})}$ & 6 \\
\hline $\mathrm{V}_{\text {int }}$ & $0.20 \mathrm{eV}$ \\
\hline initial value: $\theta$ & 0.001 \\
\hline initial value: $\mathrm{y}^{(\mathrm{A})}$ & $1 / 3$ \\
\hline initial value: $\mathrm{y}^{(\mathrm{B})}$ & $1 / 3$ \\
\hline initial value: $\mathrm{y}^{(\mathrm{C})}$ & $1 / 3$ \\
\hline
\end{tabular}

Having access to the free energy (per unit cell) of this system as a function of total coverage and site fractions (Equation 25), we are able plot this and the corresponding chemical potentials of each site type, as well, which allows us to further characterize the physicality of the models. The results for Model $\alpha$ and Model $\beta$ are shown in Figure 6. 

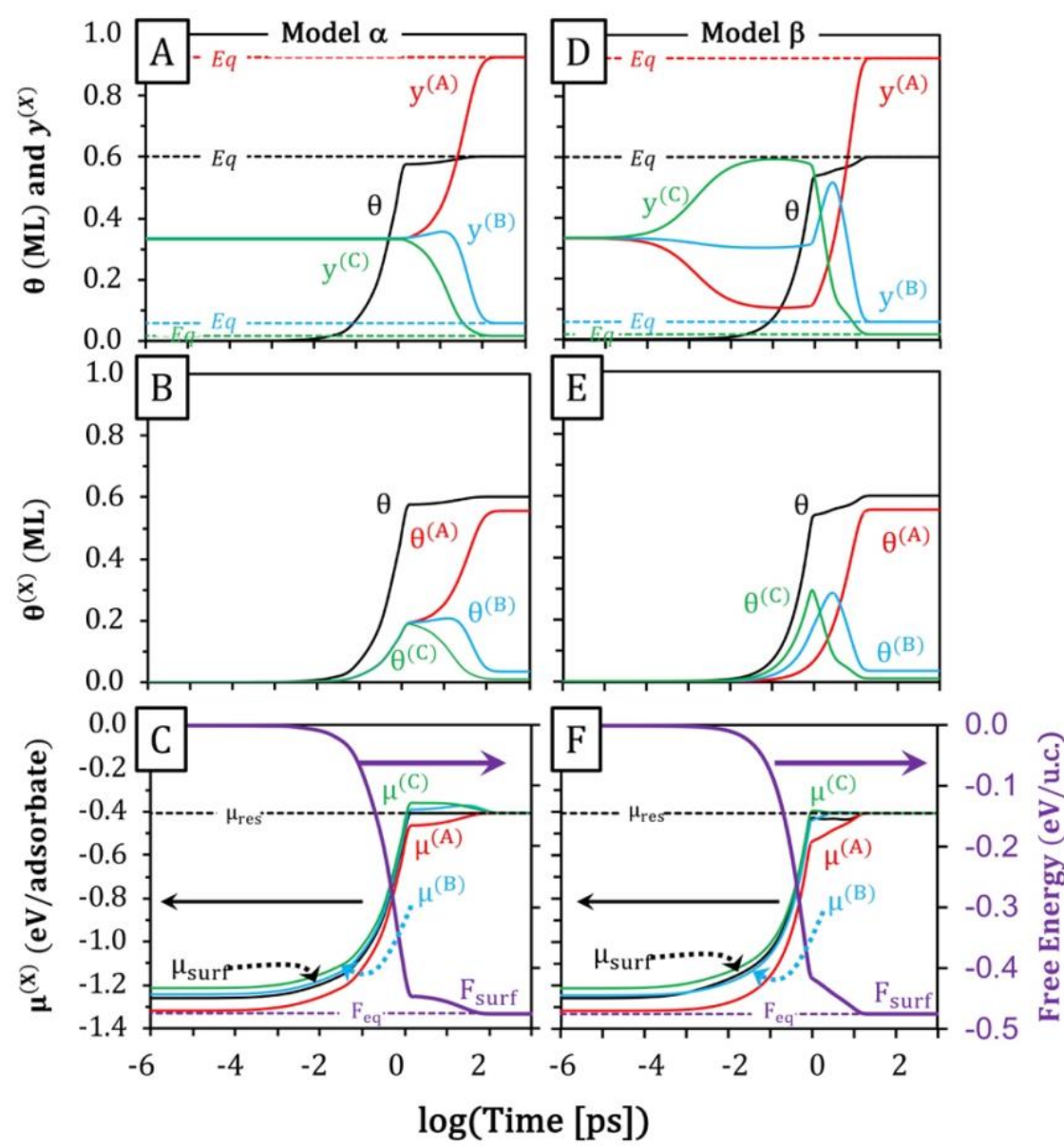

Figure 6. Solution of the (A-C) Model $\alpha$ and (D-F) Model $\beta$ MF-MKMs for Scenario 1, showing (A,D) the integrated value of the total coverage $\left(\theta\right.$, solid black line) and site fractions $A, B$, and $C\left(y^{(x)}\right.$, solid red, blue, and green lines, respectively); $(B, E)$ the site coverages $\left(\theta^{(X)}\right.$, solid red, blue, and green lines, respectively) alongside the total coverage again $(\theta$, solid black line) for comparison; and (C,F) the corresponding plots of the system's mean field thermodynamics: solid black lines are the effective desorption chemical potential ( $\left.\mu_{\text {surf }}\right)$ given in Equation 19, solid red, blue, and green lines are site type chemical potentials $\left(\mu^{(X)}\right)$, the solid purple lines are free energies on a unit cell basis. In $(A-F)$, equilibrium values as determined from minimization of the free energy (Equation 25b) and are shown as same-color horizontal dashed lines. Note that the simulation time ( $x$-axis) is shown on a log scale.

As seen in Figure 6A, integration of Model $\alpha$ produces values of $\theta$ and $\mathrm{y}^{(\mathrm{X})}$ (solid lines) that match their predicted equilibrium values once steady state is reached around $t=10^{2} \mathrm{ps}$-just as demanded by thermodynamic self-consistency. The corresponding values $\theta^{(\mathrm{X})}$ are shown in Figure $6 \mathbf{B}$, revealing the dynamic behavior of diffusion between site types. Sensibly, as coverage increases, diffusion increases toward sites that exhibit lower chemical potentials (A and B sites, see Figure 6C). However, we can see that the early kinetics are not dominated by diffusion but by adsorption, which greatly outpaces both desorption and diffusion until the coverage increases to $\sim 0.57 \mathrm{ML}$ around $\mathrm{t}=1 \mathrm{ps}$, at which point rates become comparable and we see total coverage and site fractions move toward equilibrium in tandem-with clearly different kinetics involved as the rates abruptly change. Interestingly, $0.57 \mathrm{ML}$ is the total coverage for which the equilibrium site fractions are $1 / 3,1 / 3$, and $1 / 3$ - the initial values used. This means that the system's initial kinetics are driven by its attempt to minimize the free energy at the initial site fraction values used. As (initial) adsorption rates will almost always outpace diffusion rates, this suggests initial site fractions should in general be chosen as those that minimize the free energy at the chosen initial total coverage instead of arbitrary values unless there is good reason to believe the surface starts off in an unequilibrated state. Overall, however, the kinetic behavior of Model $\alpha$ is sensible and does not display any aphysicality beyond perhaps that 
caused by initializing the system in an arbitrary manner. It takes $\sim 1.3 \mathrm{ps}$ to reach the kinetic change over from adsorption dominated to diffusion dominated at $\sim 0.57 \mathrm{ML}$, and another $\sim 100 \mathrm{ps}$ to reach equilibrium from there. Given that transition states were set at a very high value of $0.0 \mathrm{eV}$, the diffusion barriers are very large (on the order of $\sim 1.0 \mathrm{eV}$ ), and slow diffusion kinetics between site types are thus expected.

Compared to Model $\alpha$, the integration of Model $\beta$, shown in Figure 6D and Figure 6E, is markedly aphysical in that the highest chemical potential site (site $C$ see Figure $6 F$ ) is initially preferentially occupied over the other lower chemical potential sites, with the lowest chemical potential site (site A) even being preferentially depleted. This is a result of defining individual adsorption rates (Equations $30 \mathrm{~b}, 30 \mathrm{~d}$, and 30f) with transitions states that are proportional to the site type area, $\mathrm{a}_{\mathrm{S}}^{(\mathrm{X})}$, and thus to $\mathrm{n}^{(\mathrm{X})}$, which is largest for site $\mathrm{C}\left(\mathrm{n}^{(\mathrm{C})}=6\right)$. This makes the site $\mathrm{C}$ adsorption rate largest regardless of its binding strength (i.e., chemical potential). In the extreme, this suggests that a nominally inert support (e.g. $\mathrm{SiO}_{2}$ ) would experience preferential adsorbate binding to it over its supported active nanoparticles simply because the support constitutes the majority of the surface area. Arbitrary corrections could be added to correct for this, but this fact itself makes it clear that such rate expressions are flawed-supporting the validity of Equation 19. As seen in Figure 6F, this is despite the underlying thermodynamics of Model $\beta$ appearing to behave qualitatively similarly to Model $\alpha$. While a clear kinetic changeover occurs around $t=0.9 \mathrm{ps}$, similar to that seen in Model $\alpha$, this point does not correspond to the minimization of the free energy at the initial site fractions or those reached at $\mathrm{t}=\sim 0.9 \mathrm{ps}$. Instead, the change in kinetics is merely the result of $\mathrm{C}$ site adsorption coming to equilibrium with the reservoir (where its individual desorption and adsorption rates reach the same value), leaving the next highest surface area site, site $B$, to dominate adsorption until it comes to equilibrium with the reservoir, as well. We see a maximum in $\theta^{(C)}$ and $\theta^{(B)}$ (solid green and blue lines in Figure 6E, respectively) where adsorption/desorption equilibrium is reached for each site type. This suggests that only at these points, where net adsorption reaches zero, can diffusion work to "fix" the site fractions (since diffusion rates are properly defined in Model $\beta$ still). This also leads to the awkward result that once $C$ sites reach their equilibrium value, the desorption rate (now balanced by the adsorption rate) becomes greater than the adsorption rate of either A or B sites, making the existence of multiple adsorption/desorption rates even more specious. Overall, the results of Scenario 1 show the kinetic behavior of each model is significantly different despite both models exhibiting thermodynamic self-consistency (which is contrived in Model $\beta$ ).

\section{SCENARIO 2}

In this scenario, we will treat adsorbates more realistically and compare to a model MF-MKM that treats the adsorbates according to the law of mass action, assuming reactant chemical activity to be proportional to coverage/concentration of adsorbates and empty sites. While the molecular partition function of the adsorbate occupying a certain site type would typically be that of a harmonic oscillator, we will neglect this term in both models since large differences in vibrational frequencies would be required to significantly affect the sites' energetics and is outside the scope of what we wish to demonstrate here anyway. To be clear, these partition functions are important to the thermodynamics and kinetics of real systems and cannot be neglected outside this academic exercise.

Model $\alpha$ in Scenario 2 will mimic Model $\alpha$ in Scenario 1 but with the adsorbates treated as lattice gases, using a simplified mutually exclusive co-adsorption scheme for the configurational entropy term $\left(\Omega^{(\mathrm{X})}\right)$ so that the effects of site blocking can be included. Note that including site blocking restricts the maximum total or site coverages to $1 \mathrm{ML}$, which is the tacit assumption when expressing the sticking coefficient as $S_{0}(1-\theta)$ : the leading " 1 " is the maximum coverage. Depending on the adsorbate in question, such an assumed maximum coverage may be more or less realistic, but we will assume a general case here. Model $\beta$ in Scenario 2, on the other hand, will treat adsorbates according to the law of mass action, as stated previously. As opposed to that used in Model $\beta$ of Scenario 1, Model $\beta$ of Scenario 2 will exhibit a single desorption rate similar to Model $\alpha$, but without the entropy contributions of $\Omega^{(\mathrm{X})}$ since the activity will be expressed via the law of mass action. 
We will take the initial site fractions to be their equilibrium values at the initial total coverage of $0.001 \mathrm{ML}$, as the results of Model $\alpha$ in Scenario 1 suggests we should. Unsurprisingly, at such a low coverage, primarily the low chemical potential A sites are populated-a much more physical prediction than that of Model $\beta$ in Scenario 1. To show how lateral interactions can lead to a significant change in these site fractions, we will now also include specific repulsions between occupied sites of the same type (we designate these interactions as $\mathrm{V}^{(\mathrm{XX})}$ to represent the average 2-body interaction between an occupied $X$ site and other occupied $X$ sites) such that the stronger the zero-coverage binding energy $\left(\mathrm{V}^{(\mathrm{X})}\right)$ is, the more repulsions it experiences. This will drive the site fractions to change more significantly as total coverage increases.

MODEL $\alpha$

Table 3. Site Blocking Scheme for Scenario 2, Model $\alpha$.

\begin{tabular}{|c|c|c|c|}
\hline $\begin{array}{c}\text { Occupation } \\
\text { of an... }\end{array}$ & $\begin{array}{c}\text { Blocks } \\
\text { A sites }\end{array}$ & $\begin{array}{c}\text { Blocks } \\
\text { B sites }\end{array}$ & $\begin{array}{c}\text { Blocks } \\
\text { C sites }\end{array}$ \\
\hline A site & 1 & 3 & 6 \\
\hline B site & 1 & 3 & 6 \\
\hline C site & 1 & 3 & 6 \\
\hline
\end{tabular}

We define the free energy of the system again as in Equation 25, but with $q^{(A)}=q^{(B)}=q^{(C)}=1$ and the lateral interactions modified as discussed above. This gives the free energy of the surface as

$$
\mathrm{F}_{\text {surf }}=\mathrm{N}_{\text {u.c. }}\left[\mathrm{V}^{(\mathrm{A})} \theta^{(\mathrm{A})}+\mathrm{V}^{(\mathrm{B})} \theta^{(\mathrm{B})}+\mathrm{V}^{(\mathrm{C})} \theta^{(\mathrm{C})}+\frac{\mathrm{C}}{2}\left(\mathrm{~V}^{(\mathrm{AA})}\left[\theta^{(\mathrm{A})}\right]^{2}+\mathrm{V}^{(\mathrm{BB})}\left[\theta^{(\mathrm{B})}\right]^{2}+\mathrm{V}^{(\mathrm{CC})}\left[\theta^{(\mathrm{C})}\right]^{2}+\mathrm{V}_{\text {int }} \theta^{2}\right)-\mathrm{k}_{\mathrm{b}} \mathrm{T} \ln \left(\omega^{(\mathrm{A})} \omega^{(\mathrm{B})} \omega^{(\mathrm{C})}\right)\right]
$$

where $\omega^{(\mathrm{A})}, \omega^{(\mathrm{B})}$, and $\omega^{(\mathrm{C})}$ are derived from a mutually exclusive site blocking scheme (Case I in Section 2.3) where each adsorbate blocks the equivalent of one unit cell and all sites found within; this makes the maximum site coverage of each site equal to $1 \mathrm{ML}$. The explicit site blocking scheme can be found in Table 3. For $\Omega^{(\mathrm{A})}$, this produces

$$
\Omega^{(A)}=\left(\begin{array}{c}
N_{\text {u.c. }}-\mathrm{N}^{(\mathrm{B})}-\mathrm{N}^{(\mathrm{C})} \\
\mathrm{N}^{(\mathrm{A})}
\end{array}\right)\left[\mathrm{n}^{(\mathrm{A})}\right]^{\left[\mathrm{N}^{(\mathrm{A})}\right]}
$$

which, after Sterling's approximation, gives

$$
\Omega^{(A)}=\left[\frac{\left(1-\theta^{(B)}-\theta^{(C)}\right)^{\left(1-\theta^{(B)}-\theta^{(C)}\right)}}{\left(\frac{\theta^{(A)}}{n^{(A)}}\right)^{\theta^{(A)}}(1-\theta)^{(1-\theta)}}\right]^{N_{\text {u.c. }}}
$$

with $\omega^{(A)}$ being the term in square brackets. After differentiation of the free energy thus defined, the site type A chemical potential is

$$
\mu^{(A)}=V^{(A)}+c\left[V^{(A A)} \theta^{(A)}+V_{i n t} \theta\right]+k_{B} T \ln \left[\frac{\theta^{(A)}\left(1-\theta^{(A)}-\theta^{(C)}\right)\left(1-\theta^{(A)}-\theta^{(B)}\right)}{n^{(A)}(1-\theta)^{3}}\right]
$$

which is a significant deviation from an ideal $2 \mathrm{D}$ gas chemical potential where the final term in Equation 39 would simply be $\mathrm{k}_{\mathrm{B}} \mathrm{T} \ln \left[\theta^{(\mathrm{A})}\right]$ ). Note that the form of this expression prevents the total coverage, or any individual site type's coverage, from exceeding their common maximum coverage of $1 \mathrm{ML}$ due to negative logarithmic arguments outside this bound. In this way, we have introduced the saturation coverage of individual adsorbates as a thermodynamically and thus kinetically important physical quantity. An analogous set of expressions can be obtained for the other two site types.

To make diffusion kinetics in Scenario 2 slightly more realistic than in Scenario 1, we will also include a common nonzero transition state chemical potential, $\mu^{\ddagger}$, which will be inserted into each site type's diffusion rate expression. The rates of diffusion from, e.g., A sites and adsorption/desorption from the entire surface are then

$$
r_{A \rightarrow B}=r_{A \rightarrow C}=\frac{k_{B} T}{h} e^{\beta\left[\mu^{(A)}\right]}=\frac{k_{B} T}{h}\left[\frac{\theta^{(A)}\left(1-\theta^{(A)}-\theta^{(C)}\right)\left(1-\theta^{(A)}-\theta^{(B)}\right)}{n^{(A)}(1-\theta)^{3}}\right] e^{-\beta\left[\mu^{\ddagger}-\left(v^{(A)}+c\left[V^{(A A)} \theta^{(A)}+V_{i n t} \theta\right]\right)\right]}
$$




$$
\begin{aligned}
r_{\text {surf } \rightarrow \text { res }}= & S_{0}(1-\theta) \frac{\mathrm{k}_{\mathrm{B}} \mathrm{T}}{\mathrm{h}} \frac{\mathrm{a}_{\mathrm{u} . c .}}{\lambda_{\text {th }}^{2}} \mathrm{e}^{\beta\left[\mathrm{y}^{(\mathrm{A})} \mu^{(\mathrm{A})}+\mathrm{y}^{(\mathrm{B})} \mu^{(\mathrm{B})}+\mathrm{y}^{(\mathrm{C})} \mu^{(\mathrm{C})}\right]} \\
& \mathrm{r}_{\text {res } \rightarrow \text { surf }}=\mathrm{S}_{0}(1-\theta) \frac{\mathrm{P}_{\text {res }} \mathrm{a}_{\text {u.c. }}}{\sqrt{2 \pi \mathrm{k}_{\mathrm{B}} \mathrm{T}}}
\end{aligned}
$$

where $\mu^{\ddagger}$ will be defined as a single energy located at $-0.50 \mathrm{eV}$ (which produces barriers of $\sim 0.50 \mathrm{eV}$ ). We see in Equation $\mathbf{4 0}$ that diffusion rates are still proportional to their site type coverage (i.e., concentration), which is $\theta^{(\mathrm{A})}$ above, as the law of mass action would suggest. However, it is also directly proportional to the number of empty $\mathrm{B}$ and $\mathrm{C}$ sites here. This is due to site blocking: when the adsorbate leaves the $A$ site, it also increases the availability (and therefore entropy) of $B$ and $C$ sites, and so this effect is reflected in the global entropy and thus the rates. The presence of $(1-\theta)^{3}$ in the denominator is not due to site blocking and instead reflects the entropy gained by all 3 sites associated with simply making the surface emptier. Clearly, in the diffusion rates, this term cancels out at equilibrium, but removing it would distort the underlying difference in chemical potentials driving diffusion as this is a real feature of the entropy of the system. Inserting Equation 40-41 into Equation 35 produces the system of ODEs for Model $\alpha$ of Scenario 2.

\section{MODEL $\beta$}

The model that will be compared against Model $\alpha$ is based on the law of mass action, which suggests that the rate should be directly proportional to the concentration of species leaving and also proportional to the concentration of empty sites of the site type that the adsorbate is diffusing toward. To decouple effects, we will use a single desorption rate in the spirit of Equation 19 but with chemical activity determined on the basis of mass action rather than on $\Omega^{(\mathrm{X})}$. Using the site blocking scheme shown in Table 3, we therefore produce

$$
\begin{aligned}
& r_{A \rightarrow B}=\frac{k_{B} T}{h}\left(e^{-\beta\left[\mu^{\ddagger}-\left(V^{(A)}+c\left[V^{(A A)} \theta^{(A)}+V_{i n t} \theta\right]\right)\right]}\right) \theta^{(A)}\left(1-\theta^{(A)}-\theta^{(C)}\right) \\
& r_{B \rightarrow A}=\frac{k_{B} T}{h}\left(e^{-\beta\left[\mu^{\ddagger}-\left(v^{(B)}+c\left[v^{(B B)} \theta^{(B)}+V_{i n t} \theta\right]\right)\right]}\right) \theta^{(B)}\left(1-\theta^{(B)}-\theta^{(C)}\right) \\
& r_{A \rightarrow C}=\frac{k_{B} T}{h}\left(e^{-\beta\left[\mu^{\ddagger}-\left(v^{(A)}+c\left[v^{(A A)} \theta^{(A)}+V_{i n t} \theta\right]\right)\right]}\right) \theta^{(A)}\left(1-\theta^{(A)}-\theta^{(B)}\right) \\
& r_{C \rightarrow A}=\frac{k_{B} T}{h}\left(e^{-\beta\left[\mu^{\ddagger}-\left(V^{(C)}+c\left[v^{(C C)} \theta^{(C)}+V_{i n t} \theta\right]\right)\right]}\right) \theta^{(C)}\left(1-\theta^{(B)}-\theta^{(C)}\right) \\
& r_{B \rightarrow C}=\frac{k_{B} T}{h}\left(e^{-\beta\left[\mu^{\ddagger}-\left(v^{(B)}+c\left[v^{(B B)} \theta^{(B)}+V_{i n t} \theta\right]\right)\right]}\right) \theta^{(B)}\left(1-\theta^{(A)}-\theta^{(B)}\right) \\
& r_{C \rightarrow B}=\frac{k_{B} T}{h}\left(e^{-\beta\left[\mu^{\ddagger}-\left(v^{(C)}+c\left[v^{(C C)} \theta^{(C)}+V_{i n t} \theta\right]\right)\right]}\right) \theta^{(C)}\left(1-\theta^{(A)}-\theta^{(C)}\right) \\
& \mathrm{r}_{\text {surf } \rightarrow \text { res }}=\mathrm{S}_{0}(1-\theta) \frac{\mathrm{k}_{\mathrm{B}} \mathrm{T}}{\mathrm{h}} \frac{\mathrm{a}_{\text {u.c. }}}{\lambda_{\text {th }}^{2}}\left(\mathrm{e}^{\beta\left[\sum_{X} \mathrm{y}^{(\mathrm{x})}\left(\mathrm{v}^{(\mathrm{x})}+\mathrm{c}\left[\mathrm{v}^{(\mathrm{xx})} \theta^{(\mathrm{x})}+\mathrm{V}_{\text {int }} \theta\right]\right)\right]}\right) \theta \\
& r_{\text {res } \rightarrow \text { surf }}=S_{0}(1-\theta) \frac{P_{\text {res }} a_{\text {u.c. }}}{\sqrt{2 \pi \mathrm{mk}_{\mathrm{B}} \mathrm{T}}}
\end{aligned}
$$

which can be inserted into Equation 36 to yield the system of ODEs for Model $\beta$. The remaining parameters of Model $\alpha$ and Model $\beta$ of this scenario can be found in Table 4.

Just as with Model $\alpha$ in Scenario 1, we can see in Figure 7A-7C that Model $\alpha$ in Scenario 2 exhibits thermodynamic self-consistency, reproducing equilibrium values as determined by minimization of the system's total free energy. Model $\beta$ of Scenario 2 (Figure 7D-7F), on the other hand is not thermodynamically self-consistent despite the free energy being minimized to nearly the same value (see solid purple lines in Figure 7C and Figure 7F). However, the correct ordering of site fractions and nearly the same total steady state coverage is achieved, showing that the law of mass action can produce qualitatively similar results despite predicting the wrong absolute values at steady state/equilibrium (provided that a single adsorption/desorption rate pair is also used, which is the case 
here). We also now see a single kinetic regime in both models, in contrast to Scenario 1, demonstrating the important effect of starting from an equilibrated surface when initializing the system. Looking at Figure 7B and Figure 7E, we see both models exhibit a transient local maximum in the site A coverage as total coverage increases and changes the system from favoring $A$ site occupation to $C$ site occupation, which is caused by the stronger repulsions between occupied $A$ sites, eventually negating its stronger binding energy. However, since the entropic effects of site blocking are not imbedded in Model $\beta$, the final $C$ site coverage $\left(\theta^{(C)}\right)$ is only $\sim 28 \%$ greater than the final A site coverage $\left(\theta^{(A)}\right)$ when Model $\alpha$ predicts it should be $\sim 72 \%$ greater. While site coverages might not seem to be very different in absolute terms, such a discrepancy can have significant effects on surface chemistries that involve one site type but not the other. This demonstrates that great care must be taken in choosing a site blocking scheme (and free energy) that accurately reflects the physics of the real system under investigation. Overall, the results of Scenario 2 highlight the importance of site blocking and entropy and suggests that the law of mass action is not an entirely appropriate basis for diffusion rates in MFMKMs of reactive surfaces exhibiting multiple types of sites or states.

Table 4. Parameters and initial values used in MF-MKMs of Model $\alpha$ and $\beta$ of Scenario 2.

\begin{tabular}{|c|c|}
\hline Parameter & Value \\
\hline $\mathrm{T}$ & $300 \mathrm{~K}$ \\
\hline $\mathrm{P}_{\text {res }}$ & 1 bar \\
\hline $\mathrm{m}$ & $30 \mathrm{~g} / \mathrm{mol}$ \\
\hline a u.c. & $25 \AA^{2}$ \\
\hline $\mathrm{V}^{(\mathrm{A})}$ & $-1.00 \mathrm{eV}$ \\
\hline $\mathrm{V}^{(\mathrm{B})}$ & $-0.90 \mathrm{eV}$ \\
\hline $\mathrm{V}^{(\mathrm{C})}$ & $-0.85 \mathrm{eV}$ \\
\hline $\mathrm{n}^{(\mathrm{A})}$ & 1 \\
\hline $\mathrm{n}^{(\mathrm{B})}$ & 3 \\
\hline $\mathrm{n}^{(\mathrm{C})}$ & 6 \\
\hline $\mathrm{V}^{(\mathrm{AA})}$ & $0.20 \mathrm{eV}$ \\
\hline$V^{(B B)}$ & $0.10 \mathrm{eV}$ \\
\hline $\mathrm{V}^{(\mathrm{CC})}$ & $0.05 \mathrm{eV}$ \\
\hline$V_{\text {int }}$ & $0.10 \mathrm{eV}$ \\
\hline$\mu_{\mathrm{XY}}^{\ddagger}$ & $-0.50 \mathrm{eV}$ \\
\hline initial value: $\theta$ & 0.001 \\
\hline initial value: $\mathrm{y}^{(\mathrm{A})}$ & $0.9224^{a}$ \\
\hline initial value: $\mathrm{y}^{(\mathrm{B})}$ & $0.0602^{a}$ \\
\hline initial value: $\mathrm{y}^{(\mathrm{C})}$ & $0.0174^{a}$ \\
\hline \multicolumn{2}{|c|}{$\begin{array}{l}\text { a) Site Fractions that minimize the fre } \\
\text { energy at the initial total coverage, } \\
\theta=0.001 \text {. }\end{array}$} \\
\hline
\end{tabular}



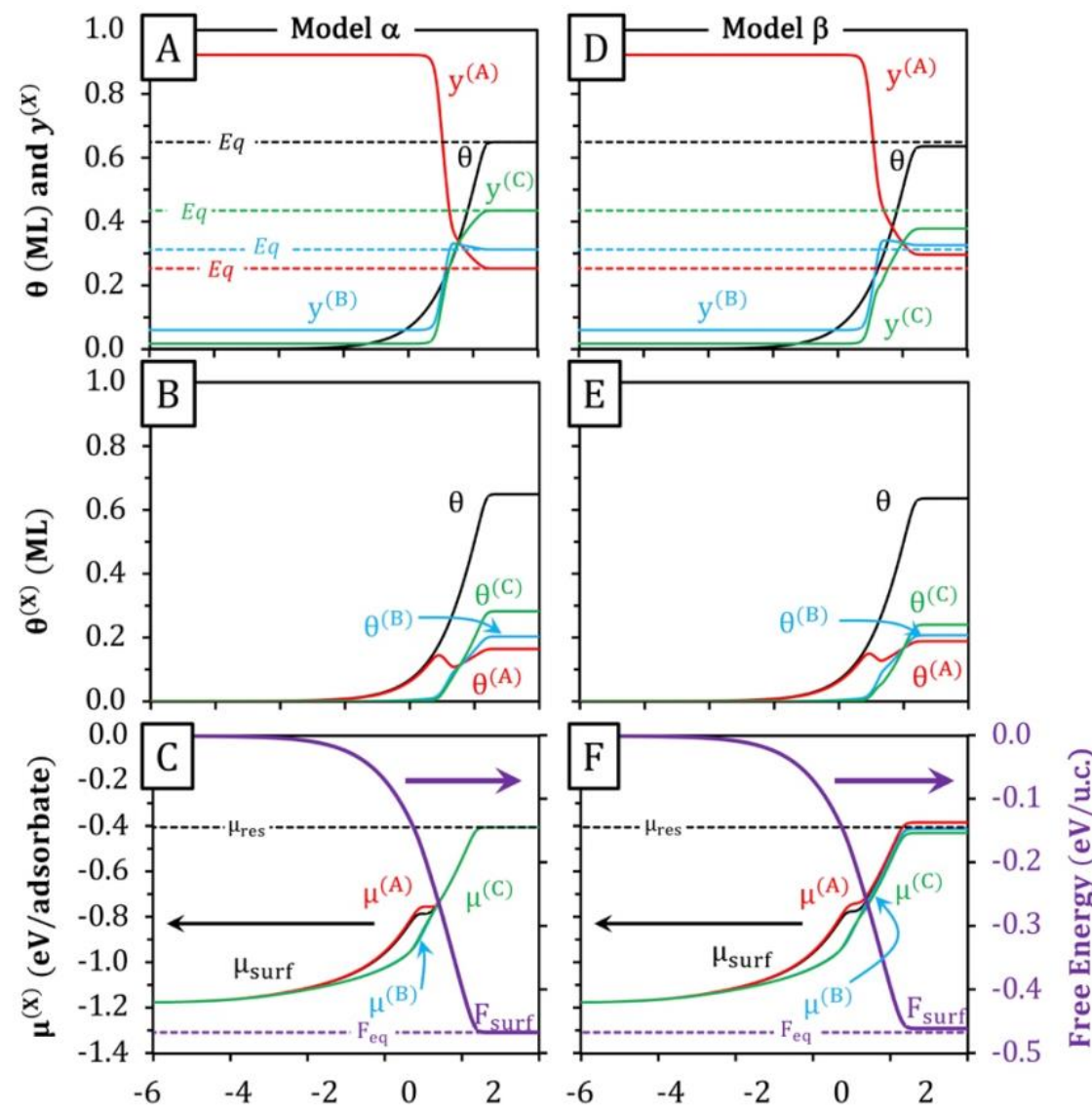

$\log ($ Time $[\mathrm{ps}])$

Figure 7. Solution of the (A) Model $\alpha$ and (B) Model $\beta$ MF-MKMs for Scenario 2: solid black lines are integrated values of the total coverage $(\theta)$, and solid red, blue, and green lines are the integrated values of site fractions $A$, $B$, and $C$, respectively. (C/D) The corresponding plots of the mean field thermodynamics: solid black lines are the effective desorption chemical potential given in Equation 19, solid red, blue, and green lines are site type chemical potentials, the solid purple lines are free energies on a unit cell basis. In (A - D), equilibrium values as determine from the free energy (Equation 37) and are shown as same-color horizontal dashed lines and denoted with same-color "eq". Note that the free energy is plotted on the same axis here. The simulation time (x-axis) is shown on a log scale and note that the range is different from that used in Figure 6.

\subsection{Response to Thermal Excitation}

We will now demonstrate the fully range of the dynamic nature of multi-site MF-MKM kinetics using Model $\alpha$ from Scenario 2. As seen in Figure 8, starting from its equilibrium solution at $300 \mathrm{~K}$, the temperature of the total system is abruptly changed (thermally excited) to $1070 \mathrm{~K}$. This causes the system to desorb nearly all its adsorbates, returning the surface to a practically clean state $(\theta=\sim 0.001$ ML, see Figure $\mathbf{8 A}$ ). The abrupt change in temperature causes the chemical potentials of each site type to come out of equilibrium (see Figure 8B) with a different chemical potential induced in each site type. At $1070 \mathrm{~K}$ at the initial coverage, site $\mathrm{C}$ has the lowest chemical potential and site $\mathrm{A}$ has the highest, and so diffusion proceeds to increase $\mathrm{C}$ site occupation. Once equilibrium between site types is reestablished, around $t=0.01 \mathrm{fs}$, diffusion proceeds toward its final equilibrium value and $\mathrm{y}^{(\mathrm{C})}$ thus goes through a maximum (and $\mathrm{y}^{(\mathrm{A})}$ goes through a minimum), eventually arriving at their steady-state equilibrium values. Once steady state is established, this procedure is then repeated, abruptly changing temperature to those shown above the panels in Figure 8, allowing the system to come to steady state again before the next temperature change is applied. The system is ultimately brought back to $300 \mathrm{~K}$ to complete the cycle and show that the same equilibrium is reestablished. Note that 
surface sites equilibrate with each other (i.e., take on the same chemical potential) much prior to the coming to equilibrium with the new reservoir chemical potential. This suggests that it could be justifiable to treat diffusion between different site types as instantaneously quasi-equilibrated in certain real systems.

The transient behavior in each temperature subsection of Figure 8 exhibits the same features mentioned above except at $100 \mathrm{~K}$. At such a low temperature and low starting coverage, diffusion kinetics are slowed to such a degree that they can no longer respond to the disparity of chemical potentials until adsorption raises the total coverage enough for lateral interactions to force diffusion to $C$ sites. This is the case at low temperatures in general: the increased coverage results in greater lateral interactions that switch favorability from $A$ sites to $C$ sites at equilibrium. At higher temperatures, the greater entropy of $C$ sites (there are six times more $C$ sites than $A$ sites in this model) also switches favorability from $A$ sites to $C$ sites at equilibrium. Thus, amongst the temperatures used here, only at $800 \mathrm{~K}$ is the total coverage and $\mathrm{C}$ site entropy low enough to maintain $\mathrm{A}$ site dominance at equilibrium. This also demonstrates that "meta stable" sites can dominate the reactive adlayer even though they are weaker binding at low coverages entirely due to the inclusion of global entropy and realistic coverage effects. Overall, these are very sensible results given the parameters used and substantiate the appropriateness of the rate expressions established here for multiple site types. It is outside the scope of this current work to explore the variations one might see by changing binding strengths, lateral interactions, number of sites, and site blocking schemes, but it should be clear that a very rich and physically meaningful kinetic space can be described using these rate expressions.
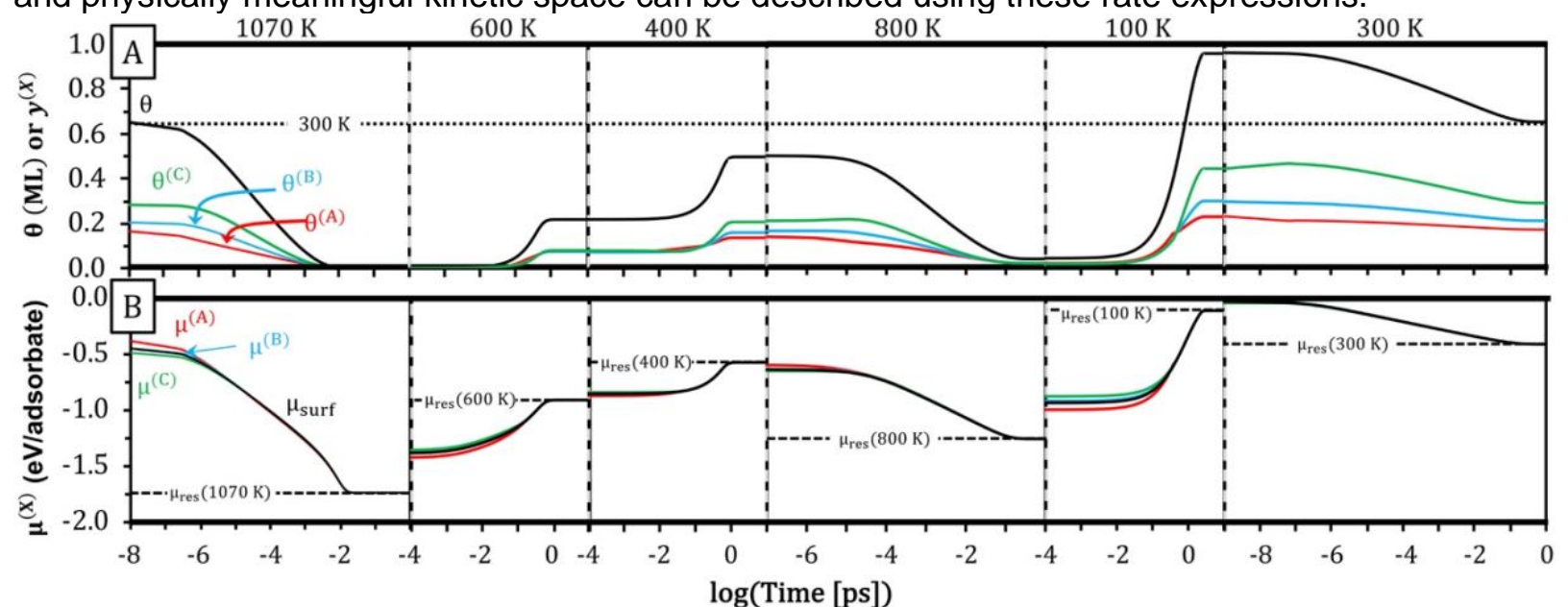

Figure 8. (A) Continuous solution of the Model $\alpha$ MF-MKM of Scenario 2 along with (B) the corresponding site type chemical potentials, $\mu^{(X)}$. The reservoir chemical potential at each temperature used to thermally excite the system is shown as a horizontal dashed line in (B). The adsorbate partial pressure is set to 1 bar throughout. The $\mathrm{x}$-axis of each temperature subsection is on its own log scale to aid the eye. Corresponding site coverages are shown in Section S4 of the SI.

\section{Discussion}

We have derived the desorption rate of a single species with multiple binding sites or states as

$$
\mathrm{r}_{\text {surf } \rightarrow \text { res }}=\frac{\mathrm{k}_{\mathrm{B}} \mathrm{T}}{\mathrm{h}} \frac{1}{\mathrm{q}_{\text {r.c. }}^{\ddagger}} \mathrm{e}^{-\beta\left[\mu_{\text {surf-res }}^{\ddagger}-\sum_{\mathrm{X}}^{\text {site types }} \mathrm{y}^{(\mathrm{x})} \mu^{(\mathrm{x})}\right]}
$$

This definition leads to an intriguing inference concerning the single transition state, $\mu_{\text {surf-res }}^{\ddagger}$, connecting the surface to the reservoir. From the point of view of thermodynamic self-consistency, $\mu_{\text {surf-res }}^{\ddagger}$ is inconsequential as it appears as a factor on both sides of any rate pair, but its effect on kinetics is significant. If desorption from each type of site is unactivated, one can imagine a common transition state occurring at the "boundary" between the reservoir and the point above the surface where the adsorbate just starts feeling the influence of the surface. Equation $\mathbf{4 3}$ would be consistent with such a model, having a single transition state chemical potential. However, for activated desorption, such a transition state is unacceptably aphysical. Thus, by analogy to Equation 19, we 
assert that the correct definition for this transition state is also a weighted sum of individual transition state chemical potentials, that is,

$$
\mu_{\text {surf-res }}^{\ddagger}=\sum_{\mathrm{X}}^{\text {site types }} \mathrm{y}^{(\mathrm{X})} \mu_{\mathrm{X}-\mathrm{res}}^{\ddagger}
$$

where $\mu_{\mathrm{X}-\text { res }}^{\ddagger}$ is, as defined in Equation $\mathbf{1 b}$, the chemical potential of the transition state associated with desorption from individual site X. Such a definition means that the desorption rate from a multisite/state MF-MKM is in fact based on a weighted sum of activation free energies, giving

$$
\mathrm{r}_{\text {surf } \rightarrow \text { res }}=\frac{\mathrm{k}_{\mathrm{B}} \mathrm{T}}{\mathrm{h}} \frac{1}{\mathrm{q}_{\text {r.c. }}^{\ddagger}} \mathrm{e}^{-\beta\left[\sum_{\mathrm{X}}^{\text {site types }} \mathrm{y}^{(\mathrm{X})} \Delta \mu_{\mathrm{act}}^{(\mathrm{X})}\right]}
$$

where

$$
\Delta \mu_{\mathrm{act}}^{(\mathrm{X})}=\mu_{\mathrm{X}-\mathrm{res}}^{\ddagger}-\mu^{(\mathrm{X})}
$$

which is a much more satisfying result from an interpretation point of view. Note that in order to ensure thermodynamic self-consistency, $\mu^{(\mathrm{X})}$ must still be properly defined.

However, it is still perhaps counterintuitive that the desorption rate from a surface (or any system) having more than one populated adsorption site (or state) should be a single rate at all and not a sum of multiple desorption rates, one for each site. This can be rationalized by considering the entire surface as one entity, which is in essence what the mean field assumption represents. When molecules of the same species enter or leave this entity, we do not have spatial resolution and so cannot assign to those molecules any specific sites directly-they must be inferred. Once the molecules enter the reservoir, molecules of the same species are identical and do not carry with them knowledge of where they came from. This of course does not mean that the rate of desorption is not physically made up of individual desorption events from individual sites. Rather, what this work demonstrates is that when a very large number of such desorption events occur, it is the average chemical potential that determines the resulting desorption rate, because again, the surface is one entity and must therefore exhibit only one chemical potential. This should not be a cause for alarm since this is all one can experimentally measure anyway. Once enough desorbing molecules reach the detector to be measurable, so many such desorption events (on the order of $10^{23}$ ) have occurred that any rate measured can only ever be an average. From this perspective, mean field models are ideal, and now that we know what is being measured (the mean chemical potential of each molecular specie's adlayer), experiments can potentially be devised to uncover the site-specific nature of the adlayers with the help of this theory and the models built around it.

Finally, we should note that a single desorption rate based on an average chemical potential is analogous to the Widom insertion method ${ }^{50}$ often used in Monte Carlo simulated desorption. When determining the desorption rate from a spatially resolved lattice, a probe molecule of the species in question is inserted into all of its available binding sites/configurations and it is the average free energy change associated with every insertion of that molecule that is used to define a single chemical potential that is inserted into a single rate expression for desorption. In this sense, the desorption rate derived here can be seen as an extension of this method to mean field microkinetics.

\section{Conclusions}

We have rigorously constructed a theoretical framework for the development of mean field microkinetic models (MF-MKMs) where adsorbates have access to more than one adsorption motif or binding site. Specifically, rate expressions have been derived from the first principles of statistical thermodynamics and a principle we refer to as "thermodynamic self-consistency", which requires that equilibrium coverages of each site, as determined by minimization of the systems total free energy, be reproduced by the MF-MKM of the same system. By comparing solutions of MF-MKMs built using these rate expressions against those built using more commonly assumed forms, we conclusively show (1) that the measured rate of desorption from a surface where multiple adsorption sites are populated cannot be a simple summation of individual desorption rates, but rather must be a single rate based on the average chemical potential of the surface adlayer, (2) that this chemical potential is dependent on the relative distribution of the available site types as well as their occupation; and (3) 
that the law of mass action is an improper basis for determining the chemical activity/coverage dependence of diffusion rates from one site to another. This latter point was touched on by Árnadóttir and co-workers, ${ }^{51}$ though in a different context. We expect that this work will provide both experimental and computational researchers the tools needed to uncover the kinetic role of multiple active sites or states in catalytic systems.

\section{Acknowledgements}

Work at WSU was primarily funded by the U.S. Department of Energy, Office of Basic Energy Sciences, Division of Chemical Sciences, Biosciences and Geosciences within the Catalysis Science program, grant DE-SC0014560. This work was also supported by the National Science Foundation Graduate Research Fellowship Program (GRFP) and Belgian American Education Foundation (B.A.E.F.). G.C., Seattle Chapter ARCS Fellow and NSF Graduate Research Fellow, gratefully acknowledges financial support from the Achievement Rewards for College Scientists foundation, and the National Science Foundation Graduate Research Fellowship Program. PNNL is a multi-program national laboratory operated for the U.S. DOE by Battelle. Work at the LCT was supported by the Flemish Research Foundation (FWO) under the Odysseus program, FWO G0E5714N. PNNL is a multi-program national laboratory operated for the U.S. DOE by Battelle. 


\section{REFERENCES}

(1) Wittreich, G. R.; Alexopoulos, K.; Vlachos, D. G. Microkinetic Modeling of Surface Catalysis. In Handbook of Materials Modeling: Applications: Current and Emerging Materials, W. Andreoni; S. Yip, Eds. Springer International Publishing: Cham, 2020; pp 1377-1404.

(2) Christiansen, M. A.; Mpourmpakis, G.; Vlachos, D. G. DFT-driven multi-site microkinetic modeling of ethanol conversion to ethylene and diethyl ether on Y-Al2O3(111). Journal of Catalysis 2015, 323, 121131.

(3) Soares, J. B. P.; Hamielec, A. E. Copolymerization of Olefins in a Series of Continuous Stirred-Tank Slurry-Reactors Using Heterogeneous Ziegler-Natta and Metallocene Catalysts. I. General Dynamic Mathematical Model. Polymer Reaction Engineering 1996, 4, 153-191.

(4) Zijlstra, B.; Broos, R. J. P.; Chen, W.; Bezemer, G. L.; Filot, I. A. W.; Hensen, E. J. M. The Vital Role of Step-Edge Sites for Both CO Activation and Chain Growth on Cobalt Fischer-Tropsch Catalysts Revealed through First-Principles-Based Microkinetic Modeling Including Lateral Interactions. ACS Catalysis 2020, 10, 9376-9400.

(5) Boudart, M. Kinetics of Chemical Processes: Butterworth-Heinemann Series in Chemical Engineering; Elsevier, 2014, p 189.

(6) Boudart, M. Two-step catalytic reactions. AlChE Journal 1972, 18, 465-478.

(7) Zhang, L.; Filot, I. A. W.; Su, Y.-Q.; Liu, J.-X.; Hensen, E. J. M. Understanding the Impact of Defects on Catalytic CO Oxidation of LaFeO3-Supported Rh, Pd, and Pt Single-Atom Catalysts. The Journal of Physical Chemistry C 2019, 123, 7290-7298.

(8) Chen, W.; Kimpel, T. F.; Song, Y.; Chiang, F.-K.; Zijlstra, B.; Pestman, R.; Wang, P.; Hensen, E. J. M. Influence of Carbon Deposits on the Cobalt-Catalyzed Fischer-Tropsch Reaction: Evidence of a TwoSite Reaction Model. ACS Catalysis 2018, 8, 1580-1590.

(9) Pestman, R.; Chen, W.; Hensen, E. Insight into the Rate-Determining Step and Active Sites in the Fischer-Tropsch Reaction over Cobalt Catalysts. ACS Catalysis 2019, 9, 4189-4195.

(10) Soares, J. B. P.; Hamielec, A. E. Deconvolution of chain-length distributions of linear polymers made by multiple-site-type catalysts. Polymer 1995, 36, 2257-2263.

(11) Kumawat, J.; Gupta, V. K. Single to Multiple Site Behavior of Metallocenes through C-H Activation for Olefin Polymerization: A Mechanistic Insight from DFT. ACS Catalysis 2020, 10, 1704-1715.

(12) Soares, J. B. P.; Abbott, R. F.; Willis, J. N.; Liu, X. A new methodology for studying multiple-site-type catalysts for the copolymerization of olefins. Macromolecular Chemistry and Physics 1996, 197, 33833396.

(13) Chen, C.; Feng, N.; Guo, Q.; Li, Z.; Li, X.; Ding, J.; Wang, L.; Wan, H.; Guan, G. Surface engineering of a chromium metal-organic framework with bifunctional ionic liquids for selective CO2 adsorption: Synergistic effect between multiple active sites. Journal of Colloid and Interface Science 2018, 521, 91-101.

(14) Soares, J. B. P. A Second Look at Modeling the Multiplicity of Active Site Types of Ziegler-Natta Catalysts with Flory's and Stockmayer's Distributions. Polymer Reaction Engineering 1998, 6, 225241.

(15) Vojvodic, A.; Nørskov, J. K. New design paradigm for heterogeneous catalysts. National Science Review 2015, 2, 140-143.

(16) Kumkaew, P.; Wu, L.; Praserthdam, P.; Wanke, S. E. Rates and product properties of polyethylene produced by copolymerization of 1-hexene and ethylene in the gas phase with (n-BuCp)2ZrCl2 on supports with different pore sizes. Polymer 2003, 44, 4791-4803.

(17) Semikolenova, N. V.; Zakharov, V. A.; Echevskaja, L. G.; Matsko, M. A.; Bryliakov, K. P.; Talsi, E. P. Homogeneous catalysts for ethylene polymerization based on bis(imino)pyridine complexes of iron, cobalt, vanadium and chromium. Catalysis Today 2009, 144, 334-340.

(18) Rohling, R. Y.; Hensen, E. J. M.; Pidko, E. A. Multi-site Cooperativity in Alkali-Metal-Exchanged Faujasites for the Production of Biomass-Derived Aromatics. ChemPhysChem 2018, 19, 446-458.

(19) Corma, A. Heterogeneous Catalysis: Understanding for Designing, and Designing for Applications. Angewandte Chemie International Edition 2016, 55, 6112-6113.

(20) Passmore, L. A.; Booth, C. R.; Vénien-Bryan, C.; Ludtke, S. J.; Fioretto, C.; Johnson, L. N.; Chiu, W.; Barford, D. Structural Analysis of the Anaphase-Promoting Complex Reveals Multiple Active Sites and Insights into Polyubiquitylation. Molecular Cell 2005, 20, 855-866. 
(21) Liu, J.; Yang, G.-P.; Jin, J.; Wu, D.; Ma, L.-F.; Wang, Y.-Y. A first new porous d-p HMOF material with multiple active sites for excellent CO2 capture and catalysis. Chemical Communications 2020, 56, 2395-2398.

(22) Li, T.-H.; Yan, M.; Liu, Y.; Huang, Z.-Q.; Chang, C.-R.; Li, J. Cooperative Catalysis by Multiple Active Centers in Nonoxidative Conversion of Methane. The Journal of Physical Chemistry C 2020, 124, 13656-13663.

(23) Song, F.; Zhang, T.; Wang, C.; Lin, W. Chiral porous metal-organic frameworks with dual active sites for sequential asymmetric catalysis. Proceedings of the Royal Society A: Mathematical, Physical and Engineering Sciences 2012, 468, 2035-2052.

(24) O'Keefe, B. J.; Breyfogle, L. E.; Hillmyer, M. A.; Tolman, W. B. Mechanistic Comparison of Cyclic Ester Polymerizations by Novel Iron(III)-Alkoxide Complexes: Single vs Multiple Site Catalysis. Journal of the American Chemical Society 2002, 124, 4384-4393.

(25) Guo, Y.; Yuan, P.; Zhang, J.; Xia, H.; Cheng, F.; Zhou, M.; Li, J.; Qiao, Y.; Mu, S.; Xu, Q. Co2P-CoN Double Active Centers Confined in N-Doped Carbon Nanotube: Heterostructural Engineering for Trifunctional Catalysis toward HER, ORR, OER, and Zn-Air Batteries Driven Water Splitting. Advanced Functional Materials 2018, 28, 1805641.

(26) Guo, J.; Yang, W.; Zhang, Y.; Gan, L.; Fan, C.; Chen, J.; Peng, Y.; Li, J. A multiple-active-site Cu/SSZ13 for NH3-SCO: Influence of Si/Al ratio on the catalytic performance. Catalysis Communications 2020, $135,105751$.

(27) Dufaud, V.; Davis, M. E. Design of Heterogeneous Catalysts via Multiple Active Site Positioning in Organic-Inorganic Hybrid Materials. Journal of the American Chemical Society 2003, 125, 9403-9413.

(28) Quan, Y.; Lan, G.; Shi, W.; Xu, Z.; Fan, Y.; You, E.; Jiang, X.; Wang, C.; Lin, W. Metal-Organic Layers Hierarchically Integrate Three Synergistic Active Sites for Tandem Catalysis. Angewandte Chemie International Edition 2020, $n / a$.

(29) Zhang, J.; Fan, H.; Li, B.-G.; Zhu, S. Effect of Catalysts Supporting on Tandem Polymerization of Ethylene Stock in Synthesis of Ethylene-1-Hexene Copolymer. Industrial \& Engineering Chemistry Research 2008, 47, 5369-5375.

(30) Sun, G.; Fuller, J. T.; Alexandrova, A. N.; Sautet, P. Global Activity Search Uncovers Reaction Induced Concomitant Catalyst Restructuring for Alkane Dissociation on Model Pt Catalysts. ACS Catalysis 2021, 11, 1877-1885.

(31) Zandkarimi, B.; Poths, P.; Alexandrova, A. N. When Fluxionality Beats Size Selection: Acceleration of Ostwald Ripening of Sub-Nano Clusters. Angewandte Chemie International Edition 2021, 60, 1197311982.

(32) Zhang, Z.; Zandkarimi, B.; Alexandrova, A. N. Ensembles of Metastable States Govern Heterogeneous Catalysis on Dynamic Interfaces. Accounts of Chemical Research 2020, 53, 447-458.

(33) Guo, H.; Poths, P.; Sautet, P.; Alexandrova, A. N. Oxidation Dynamics of Supported Catalytic Cu Clusters: Coupling to Fluxionality. ACS Catalysis 2022, 12, 818-827.

(34) Kreuzer, H. J. Theoretical Approaches to Surface Kinetics: A Perspective. Zeitschrift für Physikalische Chemie 2009, 223, 105-130.

(35) Kreuzer, H. J.; Payne, S. H.; Jakob, P.; Menzel, D. Coupled desorption and site conversion of coadsorbates: A lattice gas analysis of thermal desorption and spectroscopic data of $(\mathrm{NO}+\mathrm{O}) / \mathrm{Ru}(001)$. Surface Science 1999, 424, 36-54.

(36) Payne, S. H.; Kreuzer, H. J. Multilayer adsorption and desorption. Surface Science 1995, 338, 261278.

(37) Payne, S. H.; LeDue, J. M.; Michael, J. C.; Kreuzer, H. J.; Wagner, R.; Christmann, K. Analysis and theory of multilayer desorption: Ag on Re. Surface Science 2002, 512, 151-164.

(38) McEwen, J. S.; Payne, S. H.; Kreuzer, H. J.; Kinne, M.; Denecke, R.; Steinrück, H. P. Adsorption and desorption of $\mathrm{CO}$ on $\mathrm{Pt}(111)$ : a comprehensive analysis. Surface Science 2003, 545, 47-69.

(39) Payne, S. H.; McEwen, J. S.; Kreuzer, H. J.; Menzel, D. Adsorption and desorption of CO on Ru(0001): A comprehensive analysis. Surface Science 2005, 594, 240-262.

(40) Nielsen, J.; d'Avezac, M.; Hetherington, J.; Stamatakis, M. Parallel kinetic Monte Carlo simulation framework incorporating accurate models of adsorbate lateral interactions. The Journal of Chemical Physics 2013, 139, 224706.

(41) Ravipati, S.; d'Avezac, M.; Nielsen, J.; Hetherington, J.; Stamatakis, M. A Caching Scheme To Accelerate Kinetic Monte Carlo Simulations of Catalytic Reactions. The Journal of Physical Chemistry A 2020, 124, 7140-7154. 
(42) Savva, G. D.; Stamatakis, M. Comparison of Queueing Data-Structures for Kinetic Monte Carlo Simulations of Heterogeneous Catalysts. The Journal of Physical Chemistry A 2020, 124, 7843-7856.

(43) Stamatakis, M.; Vlachos, D. G. A graph-theoretical kinetic Monte Carlo framework for on-lattice chemical kinetics. The Journal of Chemical Physics 2011, 134, 214115.

(44) Stamatakis, M.; Vlachos, D. G. Unraveling the Complexity of Catalytic Reactions via Kinetic Monte Carlo Simulation: Current Status and Frontiers. ACS Catalysis 2012, 2, 2648-2663.

(45) Bajpai, A.; Frey, K.; Schneider, W. F. Comparison of Coverage-Dependent Binding Energy Models for Mean-Field Microkinetic Rate Predictions. Langmuir 2020, 36, 465-474.

(46) Chen, Z.; Wang, H.; Su, N. Q.; Duan, S.; Shen, T.; Xu, X. Beyond Mean-Field Microkinetics: Toward Accurate and Efficient Theoretical Modeling in Heterogeneous Catalysis. ACS Catalysis 2018, 8, 58165826.

(47) Hill, T. L. An introduction to statistical thermodynamics; Courier Corporation, 1986, p 235.

(48) Collinge, G.; Groden, K.; Stampfl, C.; McEwen, J.-S. Formulation of Multicomponent Lattice Gas Model Cluster Expansions Parameterized on Ab Initio Data: An Introduction to the Ab Initio Mean-Field Augmented Lattice Gas Modeling Code. The Journal of Physical Chemistry C 2020, 124, 2923-2938.

(49) Bray, J.; Collinge, G.; Stampfl, C.; Wang, Y.; McEwen, J.-S. Predicting the Electric Field Effect on the Lateral Interactions Between Adsorbates: O/Fe(100) from First Principles. Topics in Catalysis 2018, 61, 763-775.

(50) Widom, B. Some Topics in the Theory of Fluids. The Journal of Chemical Physics 1963, 39, 28082812.

(51) Campbell, C. T.; Sprowl, L. H.; Árnadóttir, L. Equilibrium Constants and Rate Constants for Adsorbates: Two-Dimensional (2D) Ideal Gas, 2D Ideal Lattice Gas, and Ideal Hindered Translator Models. The Journal of Physical Chemistry C 2016, 120, 10283-10297. 
TOC GRAPHIC

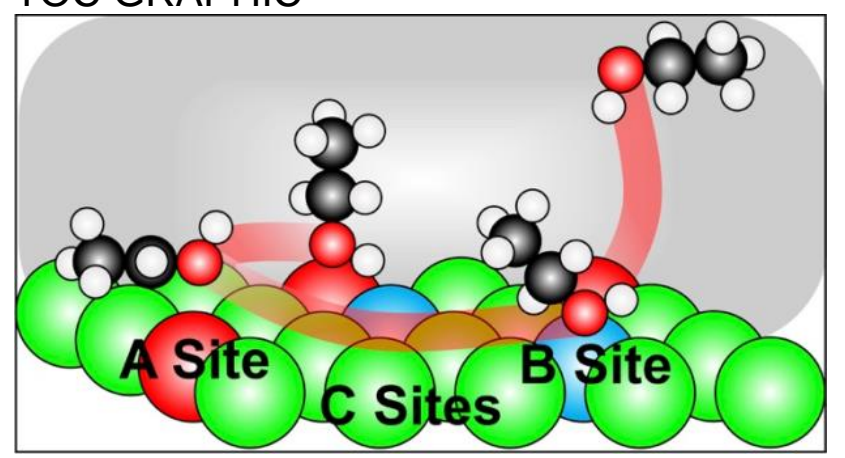

